\title{
Glibenclamide Directly Prevents Neuroinflammation by Targeting SUR1-TRPM4-Mediated NLRP3 Inflammasome Activation in Microglia
}

\section{Yihua $\mathrm{He}$}

Southern Medical University Nanfang Hospital

\section{Yuan Chang}

Southern Medical University Nanfang Hospital

\section{Yuqin Peng}

Southern Medical University Nanfang Hospital

Juan Zhu

Southern Medical University Nanfang Hospital

Kewei Liu

Southern Medical University Nanfang Hospital

Jiancong Chen

Southern Medical University Nanfang Hospital

Yongming Wu

Southern Medical University Nanfang Hospital

\section{Zhong Ji}

Southern Medical University Nanfang Hospital

Zhenzhou Lin

Southern Medical University Nanfang Hospital

\section{Shengnan Wang}

Southern Medical University Nanfang Hospital

\section{Sohan Gupta}

Southern Medical University Nanfang Hospital

\section{Nailiang Zang}

Southern Medical University Nanfang Hospital

\section{Suyue Pan}

Southern Medical University Nanfang Hospital

Kaibin Huang ( $\nabla$ hkb@smu.edu.cn )

Southern Medical University Nanfang Hospital 
Keywords: glibenclamide, SUR1-TRPM4, NLRP3 inflammasome, microglia, cardiac arrest/cardiopulmonary resuscitation, radiation-induced brain injury

Posted Date: November 2nd, 2021

DOI: https://doi.org/10.21203/rs.3.rs-980762/v1

License: (c) (1) This work is licensed under a Creative Commons Attribution 4.0 International License. Read Full License 


\section{Abstract \\ Background}

Glibenclamide (GLB) reduces brain edema and improves neurological outcome in animal experiments and preliminary clinical studies. Recent studies also suggested a strong anti-inflammatory effect of GLB, via inhibiting Nucleotide-binding oligomerization domain-like receptor containing pyrin domain 3 (NLRP3) inflammasome activation. However, it remains unknown whether the anti-inflammatory effect of GLB is independent of its role in preventing brain edema, and how GLB inhibits the NLRP3 inflammasome is not fully understood.

\section{Methods}

Sprague-Dawley male rats underwent 10-min asphyxial cardiac arrest and cardiopulmonary resuscitation or sham-operation. Wild type and Trpm4 $4^{-/-} \mathrm{C} 57 \mathrm{BL} / 6$ male mice underwent radiation-induced brain injury or sham-operation. The Trpm4 siRNA and GLB were injected to block sulfonylurea receptor 1-transient receptor potential M4 (SUR1-TRPM4) channel in rats and mice. Western blotting, quantitative real-time polymerase chain reaction, behavioral analysis, histological examination, and MRI Scanning were used to evaluate the role of GLB in preventing NLRP3-mediated neuroinflammation through inhibiting SUR1TRPM4, and corresponding neuroprotective effect. To further explore the underlying mechanism, BV2 cells were subjected to lipopolysaccharides, oxygen-glucose deprivation/reperfusion, or radiation.

\section{Results}

Here, in mice model of radiation-induced brain injury with minimal brain edema, GLB significantly alleviated neurocognitive deficit and neuropathological damage, via the inhibition of radiation-induced microglial NLRP3 inflammasome activation by blocking SUR1-TRPM4. Likewise, above neuroprotective effects were also confirmed in rat model of cardiac arrest with brain edema combined with neuroinflammation, through preventing SUR1-TRPM4-mediated NLRP3 activation. Of note, the above effects of GLB could be achieved by gene silencing or knockdown of Trpm4. In vitro, SUR1-TRPM4 and NLRP3 inflammasome were also activated in BV2 cells subjected to lipopolysaccharides, oxygen-glucose deprivation/reperfusion, or radiation, which could be blocked by GLB or 9-phenanthrol, a TRPM4 inhibitor. Importantly, activation of SUR1-TRPM4 in BV2 cells required the P2X7 receptor-mediated $\mathrm{Ca}^{2+}$ influx, which in turn magnified the $\mathrm{K}^{+}$efflux via the $\mathrm{Na}^{+}$influx-driven opening of $\mathrm{K}^{+}$channels, leading to the NLRP3 inflammasome activation.

\section{Conclusions}


These findings suggest that GLB has a direct anti-inflammatory neuroprotective effect independent of its role in preventing brain edema, through inhibition of SUR1-TRPM4 which amplifies $\mathrm{K}^{+}$efflux and promotes NLRP3 inflammasome activation.

\section{Introduction}

Glibenclamide (GLB) is a sulfonylurea that has been used for decades to treat type 2 diabetes. Sulfonylurea receptor 1-transient receptor potential M4 (SUR1-TRPM4) heteromer is assembled by integrating SUR1 and TRPM4, in which sulfonylureas like GLB could bind to SUR1 and thereby prolong the closed states of TRPM4 channel (1), a pore-forming protein nonselectively conducting monovalent cations (2). In recent years, it has been proved that GLB exerts neuroprotection on several neurologic disease models (3-6), potentially through inhibiting the SUR1-TRPM4 channel expressed in neurovascular units, and reducing consequential cell swelling, blood-brain barrier (BBB) disruption, and brain edema $(3,4)$. Encouragingly, in several clinical studies, GLB has been shown to reduce brain edema and even improve neurological function (7-9). More interestingly, even in some people who may not develop malignant brain edema $(10,11)$, sulfonylureas have shown neuroprotective effects, suggesting that they may have additional protective effects in addition to reducing brain edema. Indeed, animal studies have shown salutary roles of GLB in brain damages mainly characterized by neuroinflammation but not severe edema $(12,13)$, implying a neuroprotective mechanism of GLB apart from blocking the maladaptive influx of extracellular $\mathrm{Na}^{+}$and water mediated by SUR1-TRPM4.

Inflammasomes, the key protein complexes of the host-defense system, play a critical role in the inherent inflammatory process in response to danger-associated molecular patterns (DAMPs) released during tissue injury (14), among which the canonical inflammasome nucleotide-binding oligomerization domainlike receptor containing pyrin domain 3 (NLRP3) triggers the activation of the precursor of caspase-1 (procaspase-1), followed by the active caspase-1-mediated proteolytic cleavage of proinflammatory interleukin-1 $\beta$ (IL-1 $\beta$ ) and consequential excessive inflammation (15). Lamkanfi et al. (16) have corroborated that GLB functions upstream of NLRP3 to block NLRP3 inflammasome activation, suggesting a crucial role of GLB in restricting inflammation. However, it is unclear whether the antiinflammatory effects of GLB contribute to the complementary neuroprotection in inflammatory brain damages as mentioned above. In view of our and other researchers' findings that both SUR1-TRPM4 complex and NLRP3 inflammasome in microglia directly orchestrate the excessive neuroinflammation $(12,17)$, we assume that GLB might prevent the NLRP3 inflammasome activation in microglia and subsequent violent neuroinflammation through blocking SUR1-TRPM4, thus alleviating the neuroinflammatory injuries.

The mechanism underlying the potential link between SUR1-TRPM4 and NLRP3 inflammasome remains to be elucidated. After the priming phase initiated by ligating Toll-like receptor (TLR) with agonists such as lipopolysaccharides (LPS) (18), NLRP3 inflammasome is activated by DAMPs including adenosine triphosphate (ATP) which ligates the purinergic P2X7 receptor (P2X7R) $(19,20)$, a non-selective cation channel involved in the requisite decrease in intracellular $\mathrm{K}^{+}$for NLRP3 activation $(21,22)$. Previous 
studies have shown that opening P2X7R is not sufficient to reach the threshold of intracellular $\mathrm{K}^{+}$to engage NLRP3, in need of the cooperation with other $\mathrm{K}^{+}$channels in two-pore domain $\mathrm{K}^{+}$channel $\left(\mathrm{K}_{2 \mathrm{P}}\right)$ family and voltage-gated $\mathrm{K}^{+}$channel (Kv) family (23-25). However, the mechanism by which these channels synergistically launch the NLRP3 activation remains elusive. In consideration of the findings that GLB acts downstream of P2X7R (16), and the opening of above $\mathrm{K}^{+}$channels is regulated by the depolarized membrane potential $(26,27)$, we hypothesize that SUR1-TRPM4 functions as an intermediate link between P2X7R and downstream $\mathrm{K}^{+}$channels to gate the $\mathrm{K}^{+}$channels via conducting $\mathrm{Na}^{+}$inward current.

In the present study, we identified a neuroprotective role and mechanism of GLB in NLRP3-mediated neuroinflammation through blocking the SUR1-TRPM4 channel in microglia, independent of the previously known prevention of brain edema. Mechanistically, we revealed that the SUR1-TRPM4 complex served as a $\mathrm{K}^{+}$efflux amplifier in activating the NLRP3 inflammasome in microglia, via mediating $\mathrm{Na}^{+}$ influx, contributing to the depolarized membrane potential, and thereby gating downstream $\mathrm{K}^{+}$channels.

\section{Materials And Methods}

\section{Animals}

All animal experiments were performed in accordance with guidelines established by the Animal Care and Use Committee of Nanfang Hospital, Southern Medical University (Guangzhou, China). Male SpragueDawley rats (350-400 g) were purchased from the Experimental Animal Center of Southern Medical University. We used male Trpm4 $4^{-/-}$mice on C57BL/ 6 background (8-10 weeks old, $20-25 \mathrm{~g}$, Model Organisms Center, Shanghai, China) and wild type littermates. Wild type and $\mathrm{Trpm}^{-1-}$ mice were both confirmed by extracting and separating tail DNA in agarose gel. All animals in current study were housed in a specific pathogen-free facility under a strict 12-hour light/dark cycle with free access to food and water.

\section{Cell Lines Culture and Treatments}

Murine BV2 microglial cells and RAW264.7 macrophage cells were purchased from American Type Culture Collection (VA, USA) and cultured in high-glucose Dulbecco's modified Eagle's medium (DMEM; Gibco, NY, USA) containing 10\% fetal bovine serum (FBS; Gibco), $100 \mathrm{lU} / \mathrm{mL}$ penicillin, and $100 \mathrm{mg} / \mathrm{mL}$ streptomycin, under a humidified atmosphere of $5 \% \mathrm{CO}_{2}$ at $37^{\circ} \mathrm{C}$.

When BV2 or RAW264.7 cells reached 80 to $90 \%$ confluence, they were primed via radiation, oxygenglucose deprivation/reperfusion (OGD/R) or 3-hour LPS ( $1 \mu \mathrm{g} / \mathrm{mL}$; Sigma-Aldrich, MO, USA) incubation. LPS was dissolved in sterile water, while the equal volume of sterile water was added as control. The 0.5hour incubation with $\mathrm{NaN}_{3}$ (1 mmol/L; Sigma-Aldrich), nigericin $(10 \mu \mathrm{g} / \mathrm{mL} ; \mathrm{MCE}, \mathrm{NJ}$, USA), diazoxide (100 $\mu \mathrm{mol} / \mathrm{L}$; Sigma-Aldrich) or ATP ( $5 \mathrm{mmol} / \mathrm{L}$; Roche, Basel, Switzerland) was conducted 6 hours after the radiation, OGD, or LPS stimulation. Additionally, the following reagents were added into the medium 0.5 
hour preceding the intervention with ATP, $\mathrm{NaN}_{3}$, nigericin or diazoxide: MCC950 (10 $\left.\mu \mathrm{mol} / \mathrm{L} ; \mathrm{MCE}\right)$ against NLRP3, PAP-1 (10 nmol/L; MCE) inhibiting Kv1.3, EGTA (4 mmol/L; Sigma-Aldrich) chelating extracellular $\mathrm{Ca}^{2+}$, Brilliant Blue G (BBG) $(100 \mu \mathrm{mol} / \mathrm{L}$; Sigma-Aldrich) against P2X7R, 9-phenanthrol (9-Ph) $(50 \mu \mathrm{mol} / \mathrm{L}$; Sigma-Aldrich) against TRPM4, quinine ( $100 \mu \mathrm{mol} / \mathrm{L} ; \mathrm{MCE})$ inhibiting $\mathrm{K}_{2 \mathrm{P}}$ family, and sulfonylureas (100 $\mu \mathrm{mol} / \mathrm{L}$; Sigma-Aldrich) against SUR1 including GLB, gliclazide (GLZ) and glimepiride (GLM). Particularly, cells were pre-incubated with transcription factor inhibitors such as Mithramycin $A(2 \mu \mathrm{mol} / \mathrm{L} ; \mathrm{MCE})$ blocking specificity protein 1 (Sp-1) and JSH23 (30 $\mu \mathrm{mol} / \mathrm{L} ; \mathrm{MCE})$ against nuclear factor kappa-B (NF-KB) for 1 hour before challenged by LPS, radiation, or OGD.

\section{Solutions Used in Ion-replacement Experiments}

The preparation of solutions was in accordance with the previous study (23). Nominally $\mathrm{K}^{+}$free solution contains (in mM): $150 \mathrm{NaCl}, 2 \mathrm{CaCl}_{2}, 1 \mathrm{MgCl}_{2}, 10$ Hepes; Nominally $\mathrm{K}^{+}, \mathrm{Na}^{+}, \mathrm{Ca}^{2+}$ free solution contains (in $\mathrm{mM}$ ): $150 \mathrm{NMDG}-\mathrm{HCl}, 1 \mathrm{MgCl}_{2}, 10$ Hepes.

\section{Asphyxial Cardiac Arrest Model in Rats and Treatments}

Rats were randomly assigned to appropriate groups, and the 10-minute asphyxial cardiac arrest/cardiopulmonary resuscitation (CA/CPR) model was performed as per our previous study (28). In brief, the rats were anesthetized with isoflurane ( $4 \%$ for induction and $2 \%$ for maintenance; RWD, Shenzhen, China), followed by the orotracheal intubation with a $14 \mathrm{G}$ cannula (BD, Suzhou, China) and connection to a ventilator (RWD). Intravascular catheters (PE50; Smiths Medical, Ashford, UK) were inserted into the right femoral artery and vein for dynamic blood pressure monitoring and drug delivery, respectively. After 10-minute stabilization, rats were chemically paralyzed by IV Vecuronium $(2 \mathrm{mg} / \mathrm{kg})$, and then the ventilator was disconnected for 10 minutes, typically leading to circulatory arrest in 5 minutes, which was characterized by the cessation of arterial pulse and a decrease of mean arterial pressure (MAP) to $20 \mathrm{mmHg}$ below. At the end of 10-minute asphyxia, cardiopulmonary resuscitation was initiated by effective ventilation with $100 \%$ oxygen and intravenous administration of epinephrine $(0.01$ $\mathrm{mg} / \mathrm{kg}$ ), simultaneously accompanied by the chest compressions (200 compressions/minute). Additional doses of epinephrine $(0.02 \mathrm{mg} / \mathrm{kg})$ were given at 2-minute intervals until the return of spontaneous circulation (ROSC), as defined by an increase of MAP to $60 \mathrm{mmHg}$ above for at least 10 minutes. After spontaneous respiration recovery, rats were weaned from ventilator and extubated, but the rats that failed to ROSC within 5 minutes or were unable to be weaned from ventilator after 1 -hour observation were excluded from the continuing experiments.

GLB was dissolved in dimethyl sulfoxide (DMSO) and diluted in saline $(2.5 \mu \mathrm{g} / \mathrm{mL})$. After ROSC, GLB was administrated intraperitoneally with an initial dose of $10 \mu \mathrm{g} / \mathrm{kg}$ at 15 minutes and 4 maintenance doses of $1.2 \mu \mathrm{g}$ per 6 hours (28), whereas rats in the vehicle group received equivalent volume of DMSO and saline (Fig. S1A). Rats that underwent all procedures except asphyxial CA/CPR were used as sham control. 
The Trpm4 in vivo Ready siRNA and the universal in vivo negative control siRNA were purchased from Ambion (MA, USA). The sequences of Trpm4 siRNA were as follows: sense 5'-

CGCUAGUAGCAGCAAAUCUtt-3' and antisense 5'-AGAUUUGCUGCUACUAGCGtg-3', which downregulated the expression of TRPM4 in vivo as per our previous study (29). $500 \mathrm{pmol}$ of Trpm4 or negative control siRNA was injected into the right lateral ventricle of the rats at a rate of $0.5 \mu \mathrm{l} / \mathrm{min}$ prior to cardiac arrest.

\section{Radiation-Induced Brain Injury (RBI) Model in Mice and Treatments}

Male C57BL/6 mice weighing 20-25 g were randomly divided into different groups. The RBI model was conducted as described in the previous study (30). After anesthetization, the head of a prone mouse was placed in a radiation Geld $\left(1 \times 1 \mathrm{~cm}^{2}\right)$ from the post-canthus line to the post-aurem line. Afterward, radiation was administered with a single dose of $30 \mathrm{~Gy}$ ( $3 \mathrm{~Gy} / \mathrm{min}$ ) employing the small animal/cell X-ray MultiRad 225 irradiator (Faxitron, AZ, USA), characterized by a source-to-skin distance of $58 \mathrm{~cm}$.

According to the previous study (31), the irradiated mice were administrated $10 \mu \mathrm{g}$ of GLB $(100 \mu \mathrm{g} / \mathrm{mL})$ intraperitoneally posterior to the whole-brain radiation, followed by the maintenance dose of $10 \mu \mathrm{g}$ once daily until euthanized, whereas irradiated mice in the vehicle group received equivalent volume of DMSO and saline (Fig. S1A). The mice in the sham group received anesthetic procedures at the same time as those irradiated but not the whole-brain radiation.

\section{Cell Irradiation Model}

The BV2 cells were seeded into a 6-well plate and irradiated with a single dose of $10 \mathrm{~Gy}(1 \mathrm{~Gy} / \mathrm{min})$ using the X-ray MultiRad 225 irradiator, since earlier studies reported that $10 \mathrm{~Gy}$ was the optimal radiation dose to activate microglia (32). After irradiation, cells were returned to the $5 \% \mathrm{CO}_{2}$ incubator. The control cells received sham-irradiation. Cells or culture supernatants were collected at indicated time points postirradiation.

\section{Cell OGD/R Model}

OGD/R on BV2 cells was performed as described previously (33). The cells were cultured in the glucosefree DMEM (Gibco), and placed in a hypoxic chamber with $1 \% \mathrm{O}_{2}, 5 \% \mathrm{CO}_{2}$, and $94 \% \mathrm{~N}_{2}$ at $37^{\circ} \mathrm{C}$ for 3 hours to mimic the hypoxic-ischemic injury, followed by the restoration with high-glucose DMEM at the normoxic condition to mimic the reperfusion. Control cells were maintained in high-glucose DMEM without oxygen deprivation.

\section{Western Blotting}

Western blotting was routinely performed as previously reported (34). Rodent brain tissues, BV2 cells, and RAW264.7 cells were homogenized in RIPA lysis buffer (Beyotime, Shanghai, China) containing protease inhibitor cocktail. After denatured in loading buffer, the samples were subjected to SDS-PAGE and then transferred to PVDF membranes (Millipore, MA, USA). After blocked by $5 \%$ non-fat milk, the membranes were incubated overnight at $4^{\circ} \mathrm{C}$ with the primary antibodies as below: Mouse anti- $\beta$-actin (Proteintech, IL, 
USA), rabbit anti-GAPDH (Proteintech), rabbit anti-TRPM4 (Sigma-Aldrich), mouse anti-SUR1 (Abcam, Cambridge, UK), rabbit anti-NLRP3 (Novus, CO, USA), rabbit anti-pro-caspase-1 (Abcam), rabbit anticaspase-1 p20 (Bioss, Beijing, China), rabbit anti-precursor of IL-1 $\beta$ (pro-IL-1 $\beta$ ) (Proteintech), rabbit anti-IL$1 \beta$ p17 (Novus), rabbit anti-Kir6.1 (Abcam), and rabbit anti-Kir6.2 (Abcam), followed by the detection via the secondary antibodies (CST, MA, USA). The densities of protein blots were quantified by ImageJ software (NIH, MD, USA) and normalized to the level of $\beta$-actin or GAPDH.

\section{Measurement of Gene Expression}

The mRNA levels of SUR1, TRPM4, NLRP3, caspase-1, IL-1ß, Kir6.1, Kir6.2, $\mathrm{K}_{2 P}$ family, Kv family, and GADPH were routinely measured by quantitative real-time polymerase chain reaction (qRT-PCR) (35). Briefly, total RNA was isolated using Trizol Reagent (Thermo Fisher Scienti $\llbracket$ c, MA, USA) and reverse transcribed to cDNA with the PrimeScript ${ }^{\mathrm{TM}}$ RT Master Mix Kit (Takara, Dalian, China) according to the manufacturer's instructions. qRT-PCR was performed using the SYBR Green master mixes (Takara) and Roche LightCycler480 System. Relative changes of mRNA expression were normalized to the level of GADPH.

\section{Neurologic Function Evaluation}

The neurologic outcome of post-cardiac arrest rats was assessed at 24, 48, 72 hours and 7 days after ROSC, utilizing a scale of neurologic deficit scores (NDSs) in which 80 was considered normal, whereas 0 represented brain death (36). The total NDSs scale consisted of 7 components: general behavioral deficit, brain-stem function, motor assessment, sensory assessment, motor behavior, behavior, and seizures.

\section{Behavioral Testing}

The Morris Water Maze was carried out to evaluate short-term spatial learning and memory as described previously $(17,37)$. The water maze apparatus is a circular tank filled with opaque water (divided into four quadrants, called Q1, Q2, Q3, Q4), and a hidden platform is submerged 2-3 cm for rats (1 cm for mice) below the water surface and not visible to the rodents. Firstly, the rodents were trained to search for the platform on day 9-12 post-cardiac arrest or for 5 consecutive days after 8 weeks post-radiation at a frequency of four trials/day, orienting by referencing 3 external cues surrounding the tank. If the rodents did not find the platform in 60 seconds, they were manually placed on it for 15 seconds. Rodents' movements were tracked by TSE VideoMot2 tracking system (Bad Homburg, Germany) to record the path and latency time taken to escape from 4 randomly assigned locations. After the training termed as acquisition trial, the probe trial was performed on the following day, when the rodents were allowed 60 seconds to explore the platform which has been removed. The percentage of total time that rodents spent in the target quadrant and the number of platform location crossings were recorded and analyzed.

\section{Histological Examination}

For detecting neuronal loss and the levels of certain markers, rats were deeply anesthetized and transcardially perfused with saline on day 14 post-surgery. Brains were fixed with $4 \%$ paraformaldehyde (PFA) overnight and embedded in paraffin, and the 4- $\mu \mathrm{m}$-thick coronal slices located at $3.5 \mathrm{~mm}$ posterior 
to bregma were stained with cresyl violet (Beyotime) and then observed under optical microscope (Olympus, Tokyo, Japan). Viable neurons in the hippocampal CA1 region had the characteristics of visible nucleus and intact cytoplasm with discernable Nissl staining, while those shrunken cell bodies surrounded by empty space were regarded as dead neurons.

Immunohistochemistry of hippocampus CA1 region from rats was conducted on day 14 post-surgery by incubation with antibodies against neuronal nuclei (NeuN; CST) for neurons, microtubule-associated protein 2 (MAP-2; Sigma-Aldrich) for dendrites, glial fibrillary acidic protein (GFAP; Abcam) for astrocytes, ionized calcium-binding adapter molecule-1 (Iba-1; Novus) and CD68 (Novus) for microglia. After the immunoperoxidase staining via secondary antibodies, the slides were counterstained with hematoxylin. In each brain section, 3 fields were randomly examined. The relative intensity of MAP-2 staining and the number of NeuN-, GFAP-, Iba-1- or CD68-positive cells in hippocampus CA1 region, were quantified using Image J software.

For immunofluorescence, rats or mice were euthanized after 12 hours post-cardiac arrest, 1 week or 8 weeks post-radiation, respectively. Following fixation with 4\% PFA, brains were immersed in 15\% and 30\% sucrose at $4^{\circ} \mathrm{C}$ for cryoprotection. With regard to the BV2 cells, they were fixed with $2 \%$ PFA for 10 minutes at room temperature. The slides of brain sections and cells were incubated with the following primary antibodies: rabbit anti-TRPM4, mouse anti-SUR1, mouse anti-CD68, goat anti-lba-1, rabbit antiGFAP (Proteintech), and rabbit anti-Doublecortin (DCX; Abcam) for immature neurons. Afterwards, the slides were washed and detected with appropriate Alexa Fluor dye of secondary antibodies preceding counterstaining with DAPI in the dark. When immunofluorescent co-localization was employed, the pairs of primary or secondary antibodies were incubated simultaneously. Finally, fluorescent signaling was observed under confocal microscope (Olympus) and analyzed using ImageJ software.

\section{Caspase-1 Activity Detection}

BV2 cells were seeded in 96-well plates, of which the activated caspase-1 was evaluated utilizing a FAMFLICA detection kit (Immunochemistry Technologies, MN, USA) as described previously with some adjustments (38). The fluorescent probe FAM-YVAD-FMK (FLICA) was employed to irreversibly label in situ activated caspase- 1 in living cells, and the green fluorescent signal directly reflected the caspase-1 activity at the time the reagent was added. Briefly, following the reconstitution with DMSO, FLICA was diluted with phosphate buffered saline (PBS), added to each sample and incubated for 1 hour prior to the nuclear staining with Hoechst. FLICA excited from 490 to $495 \mathrm{~nm}$ and emitted from 515 to $525 \mathrm{~nm}$. The images were captured with a fluorescence microscope and the fluorescence intensity of cleaved caspase1 was quantified by the multiscan spectrum (BMG, Offenburg, Germany).

\section{Measurement of Intracellular $\mathrm{Ca}^{2+}$ and Membrane Potential}

The procedure was established as described previously $(39,40)$. Experiments were typically conducted using BV-2 cells seeded into 96-well plates and allowed to adhere overnight. After treatment with LPS and indicated antagonists, the cultures were washed three times in buffer and loaded for 30 minutes in the 
dark at $37^{\circ} \mathrm{C}$, with $10 \mu \mathrm{mol} / \mathrm{L}$ of either cell-permeable $\mathrm{Ca}^{2+}$ fluorescent indicator Fluo-4AM (Beyotime) or membrane potential indicator Dioc5 (Abbkine, CA, USA). Cells were then washed again in buffer and all experiments were performed at room temperature in the dark. Using the multiscan spectrum, emission intensity was measured up to 15 minutes, and the decline in Dioc5 fluorescent intensity at $430 \mathrm{~nm}$ represents the depolarization of membrane potential. Stimulating drugs including ATP, diazoxide and nigericin were added 30 seconds after the first measurement.

\section{Measurement of Intracellular $\mathrm{K}^{+}$and $\mathrm{Na}^{+}$}

This procedure was routinely performed as previously reported (41). The BV-2 cells were seeded into 96well plates to adhere overnight. After treatment with LPS and indicated antagonists, the cells were loaded with either potassium- or sodium-sensitive fluorescent dyes, PBFI-AM ( $5 \mu \mathrm{mol} / \mathrm{L}, \mathrm{Abcam})$ or SBFI-AM ( 5 $\mu \mathrm{mol} / \mathrm{L}, \mathrm{Abcam})$, which were freshly prepared by combining with equal volume of $25 \%$ Pluronic F-127 (Solarbio, Beijing, China) for 40 minutes at $37^{\circ} \mathrm{C}$. The change in $340 \mathrm{~nm} / 380 \mathrm{~nm}$ ratio that represents the alteration of intracellular $\mathrm{K}^{+}$or $\mathrm{Na}^{+}$was analyzed with the multiscan spectrum. Stimulating drugs including ATP and diazoxide were added 30 seconds after the first measurement.

\section{Measurement of Intracellular ATP}

Cells were cultured in a 96-well plate and allowed to grow overnight. After treatment, the intracellular ATP level was measured using CellTiter-Glo luminescent cell viability assay kit (Promega, WI, USA) as previously reported (42). The luminescence was measured to calculate the ATP concentration using the standard curve.

\section{Co-immunoprecipitation}

This assay was performed in line with our previous study (17). BV2 and RAW264.7 cells were lysed in moderate lysis buffer (Genstar, Beijing, China) containing phenylmethylsulfonyl fluoride. After centrifuged at $14,000 \mathrm{~g}$ for 15 minutes, the supernatant was obtained and quantified, of which the portion containing $500 \mu \mathrm{g}$ protein was immunoprecipitated with $1 \mu \mathrm{g}$ rabbit anti-TRPM 4 or mouse anti-SUR1 under rotation overnight at $4^{\circ} \mathrm{C}$. Following the rotary incubation with $30 \mu \mathrm{l}$ protein $\mathrm{A} / \mathrm{G}$ magnetic beads (Bimake, TX, USA) for another 20 minutes at room temperature and subsequent magnetic separation, the complexes were washed 5 times, and resuspended in $40 \mu$ loading buffer prior to denatured at $100^{\circ} \mathrm{C}$. Finally, the protein complexes were subjected to Western blotting as described above, and the employed antibodies were as follows: rabbit anti-TRPM4, mouse anti-SUR1, rabbit anti-Kir6.1, and rabbit anti-Kir6.2. Whole cell lysates were used as an input control, and homophytic IgG was used as a negative control.

\section{MRI Scanning}

The 7.0T nuclear magnetic resonance scanner (Bruker Biospin, Ettlingen, Germany) was utilized to scan the consecutive $0.5-\mathrm{mm}$ thick mice brain transverse sections. Mice were anesthetized with isoflurane (5\% induction, 1-2\% maintenance) before the prostration on a custom-made holder with strapping to minimize head motion. Transverse T2-weighted images were obtained using a 2-dimensional turbo spin echo sequence (repetition time/echo time, $1600 \mathrm{~ms} / 125 \mathrm{~ms}$; flip angle, $90^{\circ}$; field of view, $40 \mathrm{~mm}$; matrix, 
200/400 r; echo planar imaging factor, 1 ; turbo spin echo factor, 10; number of signal averages, 14; total scan time, $11 \mathrm{~min}$ ) (30), ranging from the superior of the parietal lobe to the inferior of the temporal lobe. The size of lateral ventricles was analyzed via ImageJ software.

\section{Statistical Analysis}

All data were presented as means \pm SD or medians and 25th to 75th percentiles (NDSs). Continuous data were analyzed with one-way ANOVA followed by Tukey's post hoc multiple comparison tests, or as indicated. NDSs were compared using Mann-Whitney $U$ test. The data of escape latency in the water maze test were analyzed with repeated-measures ANOVA comprising treatment groups, time points, and groupsxtime interaction, followed by Tukey's post hoc multiple comparison tests. SPSS 20.0 (IBM, NY, USA) and GraphPad Prism 8.0 (GraphPad, CA, USA) were used for statistical analyses. $P<0.05$ was considered statistically significant.

\section{Results}

\section{GLB prevents NLRP3 inflammasome activation in microglia after brain radiation through blocking SUR1-TRPM4}

To address whether the anti-inflammatory neuroprotection of GLB is independent of mitigating brain edema $(3,4)$, we teased apart these two contributions by using RBI model characterized by chronic neuroinflammation involving NLRP3 inflammasome activation in microglia $(43,44)$, but almost no BBB damage and brain edema in acute phase (Fig. S2). Mice undergoing RBI modeling $(n=115)$ or sham operation ( $n=45)$ were randomly assigned to appropriate groups for different experimental purposes (Fig. S1B). Notably, no hypoglycemia was detected and there was also no significant difference in blood glucose level among the 4 experimental groups at baseline and at indicated time points after whole-brain radiation (Table S1).

We first verified whether NLRP3 inflammasome activation occurred during the acute phase following radiation, by measuring the levels of NLRP3, caspase-1 p20 and IL-1 $\beta$ p17 fragments. The results demonstrated that the levels of NLRP3, pro-caspase-1, and caspase-1 p20 from irradiated mice whole brain were significantly elevated at 6 hours and plateaued for at least 7 days after radiation, while the levels of pro-IL-1 $\beta$ and IL-1 $\beta$ p17 peaked on day 1 after radiation, followed by a gradual decline until day 7 when the post-radiation levels remained remarkably augmented in comparison with the sham group (Fig. 1A-B). These changes implied that the whole-brain irradiation accounted for the activation of NLRP3 inflammasome.

Interestingly, the protein levels of SUR1 and TRPM4 were also significantly upregulated after whole-brain radiation, and the trends were consistent with that of the NLRP3 inflammasome. Besides, fluorescent staining of mice hippocampus performed on day 7 post-radiation showed that the distribution of SUR1 was highly consistent with that of TRPM4, and TRPM4 localized massively in CD68-positive cells (Fig. 1C), suggesting the radiation-induced assembly of SUR1-TRPM4 complex mainly in microglia. To 
further characterize the possible causality of expression changes in above molecules, the levels of inflammasome-related proteins were tested following genetic silencing of Trpm 4 or pharmacological blockade of the SUR1-TRPM4 channel by GLB (Fig. 1D-E). As expected, we observed that the levels of caspase-1 p20 and IL-1 1 p 17 were dramatically augmented on day 7 post-radiation but obviously recovered in the presence of GLB or in $T r p m 4^{-/-}$mice. In sum, the above results elucidate that brain radiation fuels the activation of NLRP3 inflammasome during the acute phase, which can be reversed by GLB through blocking the SUR1-TRPM4 complex in microglia.

\section{Inhibiting SUR1-TRPM4 channel with GLB improves the brain atrophy and cognitive impairment after brain radiation}

To determine whether blockage of SUR1-TRPM4 complex exerted neuroprotection on RBI, we conducted MRI scanning to evaluate the severity of brain atrophy and the area of lateral ventricles of indicated groups 8 weeks post-radiation. As illustrated in Fig. 2A, the lateral ventricles were prominently enlarged in irradiated mice compared to the sham group, but the dilated lateral ventricles were partly restored in the irradiated $\mathrm{Trpm}^{-/-}$mice with or without GLB, or the irradiated wild type mice treated with GLB, indicating that the radiation-induced brain atrophy is mitigated via closing the SUR1-TRPM4 channel with GLB.

The mice above were next used to perform Morris Water Maze test. During the acquisition trial, the escape latency $(F=98.459, P=0.000)$ and the distance to reach platform $(F=67.979, P=0.000)$ became progressively shorter in all groups over time (Fig. 2B), and repeated-measures ANOVA revealed that the escape latency $(F=43.511, P=0.000)$ and the distance to reach platform $(F=46.882, P=0.000)$ differed significantly among the groups, with no significant interaction between groups and time points. Besides, Tukey's post hoc test showed that pharmacological or genetic inhibition of SUR1-TRPM4 substantially shorted the latency and distance in finding the hidden platform in the last 2 training days. Likewise, the frequency of crossing the platform area in the probe trial was increased when the SUR1-TRPM4 complex was inhibited, in comparison with the irradiated placebo-control mice (Fig. 2C-D). Overall, these findings render the SUR1-TRPM4 complex as a key executioner of the post-radiation brain atrophy and cognitive impairment, suggesting an anti-inflammatory neuroprotection of GLB independent of reducing brain edema.

\section{Targeting SUR1-TRPM4 with GLB ameliorates neuropathologic injury in RBI model}

To assess the role of GLB in alleviating the radiation-induced neuropathologic injury, we conducted immunofluorescence 8 weeks post-radiation to detect the activation of microglia and astrocytes, which orchestrates the post-radiation chronic neuroinflammation and inhibits the maturation of neural precursor cells $(44,45)$. Immunofluorescent staining for Iba-1 expressed in mice hippocampal DG region and cortex showed significantly more Iba-1-positive cells after radiation, compared to the sham controls (Fig. 2E, 2G). However, Iba-1-positive cells were dramatically reduced in the irradiated $T r p m 4^{-1-}$ mice in the presence or absence of GLB, or irradiated wild type mice with GLB. Likewise, intervening in SUR1-TRPM4 
complex prevented the upward tendency of GFAP-positive cells in hippocampus after radiation. Moreover, staining for DCX in hippocampal DG region reflected that more viable DCX+ immature neurons were reserved by abrogating SUR1-TRPM4 channel in RBI model (Fig. 2F-G). Taken together, these results indicate that occluding SUR1-TRPM4 with GLB provides salutary effects in alleviating neuropathological injury after brain radiation, further implying a complementary neuroprotection attributed to the antiinflammatory effects of GLB.

\section{Blockage of SUR1-TRPM4 by GLB prevents NLRP3 inflammasome activation in microglia after cardiac arrest}

To validate the generality of the above results, we next examined whether the activation of NLRP3 inflammasome could be dampened by GLB through interfering with the SUR1-TRPM4 channel in a rat model of CA/CPR with brain edema and drastic neuroinflammation $(17,46)$. Rats undergoing 10-minute CA/CPR modeling $(n=58)$ or sham operation $(n=15)$ were randomly assigned to appropriate groups for different experimental purposes (Fig. S1B). 11 rats failed to ROSC and were randomly distributed to the vehicle/control group (6/32) and the GLB/siRNA group (5/26), indicating that no significant difference in the success rates of CA/CPR was observed among groups. For resuscitated animals, 4 rats ( 2 in the vehicle/control group, 2 in the GLB/siRNA group) were unable to be weaned from ventilator, and there was no significant difference in the fail rate of removing ventilator among these groups. The time from asphyxia to cardiac arrest, the time required for ROSC, the epinephrine usage, and physiological variables including MAP, heart rate, and rectal temperature were all comparable among experimental groups (Table S2). There were also no statistical differences in laboratory results including $\mathrm{PaCO}_{2}, \mathrm{PaO}_{2}, \mathrm{PH}$, and blood glucose level at baseline or after ROSC among these groups (Table S3).

Sampling at 12 hours post-cardiac arrest was determined according to our previous studies $(17,47)$, which showed that the SUR1 and TRPM4 protein levels significantly increased from 6 to 24 hours after CA/CPR, accompanied by concurrent NLRP3 inflammasome activation. In this study, the levels of NLRP3, caspase- 1 p20, and IL-1 $\beta$ p17 from hippocampus and cortex of rats were elevated at 12 hours after CA/CPR, but reversed by pharmacological inhibition of SUR1 with GLB and gene knockdown of Trpm4 (Fig. 3A-D), implying that obstructing SUR1-TRPM4 might disturb the activation signal required for NLRP3 inflammasome. However, GLB could not suppress the upregulated SUR1 and TRPM4 at 12 hours postcardiac arrest (Fig. 3A, 3C), suggesting that GLB might inhibit the transport activity of this specific channel rather than reduce its expression as per our previous study (28). As determined by the highly consistent immunofluorescent location in the hippocampus under confocal microscopy, SUR1 showed a tight interplay with TRPM4 at 12 hours after CA/CPR, and TRPM4 was also massively localized in cells expressing CD68 (Fig. 3E), collectively depicting the formation of the complex mainly in microglia. Together, these results further support the generality that GLB suppresses NLRP3 inflammasome activation by blocking the opening of SUR1-TRPM4 channels in microglia.

\section{Inhibiting SUR1-TRPM4 channel with GLB improves neurological function after CA/CPR}


We further explored whether blockage of the SUR1-TRPM4 channel with GLB improved neurologic outcomes in CA/CPR modeling rats. We used the NDSs to assess the neurologic function after CA/CPR and observed that rats in the vehicle group presented lower NDSs at 24, 48 and 72 hours after ROSC than those in the GLB group, without prominent difference on day 7 (Fig. 3F, Fig. S3A), manifesting that the neurologic deficits caused by CA/CPR were alleviated by GLB.

We also employed the Morris Water Maze test to gauge the effect of GLB on short-term spatial learning and memory ability after cardiac arrest. During the hidden-platform training on day 9-12 after surgery, rats in the sham, vehicle, and GLB groups all showed a gradual decline in the latency to find the hidden platform over time $(F=65.183, P=0.000)$ (Fig. 3G). Besides, repeated-measures ANOVA revealed that escape latency differed significantly among the groups $(F=14.294, P=0.000)$, with no significant interaction between groups and time points $(F=1.375, P=0.241)$ (Fig. 3G). Tukey's post hoc test showed that GLB substantially rescued the extended latency caused by CA/CPR on day 10-12 (Fig. 3G). In the probe trial of the Morris Water Maze test on day 13 after surgery, the frequency of crossing the platform area was decreased in the vehicle group compared with the sham group, whereas the propensity to reduction was largely reversed by GLB (Fig. 3H, Fig. S3B). Likewise, rats administrated with GLB spent markedly more time searching in the platform quadrant (Q3) in comparison with vehicle rats (Fig. $3 \mathrm{H}$, Fig. S3B). These results collectively demonstrate that inhibiting SUR1-TRPM4 with GLB provides persistent neuroprotection and mitigates the spatial learning and memory deficiency derived from cardiac arrest.

\section{GLB ameliorates neurological injury after CA/CPR through targeting SUR1-TRPM4 channel}

To assess the profound effect of GLB on the histological damage caused by cardiac arrest, all the rats involved in the Morris Water Maze test were euthanized on day 14 after CA/CPR to conduct histological examination. The results of Nissl staining unraveled that CA/CPR elicited neuron loss in hippocampal CA1 region, a susceptible region to ischemia, whereas more viable neurons were reserved by GLB (Fig. S3C-D). Consistently, GLB-treated rats exhibited more NeuN+ neurons in hippocampal CA1 region than vehicle-treated rats (Fig. 3l-J). We also set out to evaluate the injury to dendrite by immunostaining for MAP-2. As expected, GLB partially recovered the extensive loss of dendrites caused by CA/CPR in hippocampal CA1 region (Fig. 3I-J). Neuron loss is usually accompanied by glial activation to clean up the cell debris. Herein we carried out the Iba-1, CD68, and GFAP immunohistochemical staining to verify the activation of microglia and astrocytes. As illustrated in Fig. 3I-J, dramatic increment of Iba-1-positive, GFAP-positive, and CD68-positive cells appeared in the post-cardiac arrest hippocampal CA1 region when compared to the sham controls. However, the activated glial cells were significantly reduced in the presence of GLB, as evidenced by the decreased immunoreactivities of Iba-1, CD68, and GFAP. Overall, the findings suggest that occluding SUR1-TRPM4 complex with GLB mitigates CA/CPR-induced neuropathological injury. 


\section{Assembly of SUR1-TRPM4 complex and NLRP3 inflammasome occurs in BV2 cells challenged with various injurious stimuli}

To further explore the connection between SUR1-TRPM4 complex and NLRP3 inflammasome, we first sought to examine the expression of key components of SUR1-TRPM4 complex and NLRP3 inflammasome in LPS-, radiation-, and OGD/R-primed BV2 cells, in which the latter two mimicked in vivo $\mathrm{RBI}$ and CA/CPR model respectively. As shown in the heatmaps (Fig. 4A), the mRNA levels of SUR1 (encoded by $A b c c 8$ ), TRPM4, NLRP3, caspase-1, and IL-1 $\beta$ were remarkably elevated in post-modeling cells, but reversed by inhibiting Sp-1 and NF-KB with Mithramycin A and JSH23, suggesting the engagement of the two transcriptional factors in the priming stage of NLRP3 activation $(48,49)$. Besides, no alteration was found in the levels of Kir6.1 and Kir6.2 (encoded by KcnJ8 and KcnJ11 respectively), which functioned as the components of $\mathrm{K}_{\text {ATP }}$ (SUR1/2-Kir6.x) channel, another specific target of sulfonylureas. Consistently, the massively increased protein levels of SUR1, TRPM4, and NLRP3 after modeling were determined by immunoblotting (Fig. 4B-C). Additionally, the fluorescence in Fig. 4D showed the obvious formation of SUR1-TRPM4 channel after modeling, as evidenced by the highly consistent localization of SUR1 and TRPM4.

Afterwards, the increment of caspase-1 activity was reflected by the higher fluorescent intensity and increased caspase-1 p20 in post-modeling BV2 cells treated with ATP (Fig. 4E-H). Likewise, the ATPinduced release of IL-1 $\beta$ into supernatant was also observed via Western blotting. Nevertheless, the increased caspase- 1 p20 and supernatant IL-1 $\beta$ was eliminated through intervening in NLRP3 by MCC950 (Fig. 4G-H), suggesting that ATP mainly activates canonical NLRP3 inflammasome rather than nonNLRP3 inflammasomes. Taken together, these findings indicate the tight relation of SUR1-TRPM4 but not $\mathrm{K}_{\text {ATP }}$ channel with NLRP3 inflammasome activation in response to ATP in microglia challenged with excessive inflammation, radiation, or ischemia-reperfusion injury, further suggesting the critical role of SUR1-TRPM4 in NLRP3 activation in vivo.

\section{SUR1-TRPM4 channel deficiency inhibits the activation of NLRP3 inflammasome in BV2 cells}

To further investigate the role of SUR1-TRPM4 channel in ATP-induced NLRP3 activation, we evaluated the expression of relevant molecules in ATP-treated BV2 cells challenged with LPS, when exposed to the concentration gradient of SUR1 or TRPM4 inhibitors. The results revealed that the NLRP3 inflammasome activation was almost abolished through dampening TRPM4 by $50 \mu \mathrm{mol} / \mathrm{L}$ of $9-\mathrm{Ph}$, as evaluated by the level of caspase-1 p20 and supernatant IL-1 $\beta$ returning to baseline (Fig. 5A). As indicated in Fig. 5B-D, the sulfonylureas including GLB, GLZ and GLM also inhibited NLRP3 inflammasome activation in a concentration-dependent manner. Likewise, ATP-induced NLRP3 activation was diminished by $50 \mu \mathrm{mol} / \mathrm{L}$ of 9-Ph and $100 \mu \mathrm{mol} / \mathrm{L}$ of sulfonylureas in BV2 cells radiation and OGD/R models (Fig. 5E-F). In sum, closing the SUR1-TRPM4 channel with inhibitors such as GLB effectively prevents ATP-induced NLRP3 
inflammasome activation in microglia challenged with excessive inflammation, radiation, or ischemiareperfusion injury, in consistency with the results in CA/CPR and RBI models.

\section{The activation of SUR1-TRPM4 in BV2 cells involves upstream P2X7R-mediated $\mathrm{Ca}^{2+}$ influx}

We next set out to determine the electrophysiological mechanism by which the activated SUR1-TRPM4 channel delivered the activation signal to NLRP3 inflammasome. As assessed by the level of caspase-1 p20 subunit and released IL-1 $\beta$, LPS-primed BV2 cells challenged with ATP showed apparent NLRP3 activation, whereas the activation was largely blunted due to the presence of $B B G$, a specific antagonist of P2X7R (Fig. 6A-B). Moreover, we observed a marked increase of NLRP3 inflammasome activation in LPS-treated BV2 cells with diazoxide opening SUR1-regulated channels, comparable to what was observed posterior to ATP incubation. The difference was that BBG could not eliminate caspase-1 cleavage and IL-1 $\beta$ release, but 9-Ph or GLB could, collectively demonstrating that SUR1-TRPM4 complex acts downstream of P2X7R. Thereafter, we found that EGTA depletion of extracellular $\mathrm{Ca}^{2+}$ was responsible for the inhibition of NLRP3 activation in the LPS-primed BV2 cells incubated with ATP, but the activation of NLRP3 inflammasome after diazoxide treatment showed no relation to extracellular $\mathrm{Ca}^{2+}$ concentration (Fig. 6C-D). We further detected the change in intracellular $\mathrm{Ca}^{2+}$ after various stimulations and interventions (Fig. 6E), and corroborated that the intracellular $\mathrm{Ca}^{2+}$ was significantly elevated given the stimulation with LPS and ATP, but was largely reversed when blocking the P2X7R by BBG or depleting extracellular $\mathrm{Ca}^{2+}$ by EGTA, illustrating that ATP-induced opening of P2X7R activates SUR1-TRPM4, through increasing intracellular $\mathrm{Ca}^{2+}$, the critical condition for TRPM4 channel opening (50).

\section{Lack of SUR1-TRPM4 channel suppresses the NLRP3 inflammasome activation mediated by the ATP decrease in BV2 cells}

Nigericin, a $\mathrm{K}^{+}$ionophore, elicits the NLRP3 inflammasome activation involving increased $\mathrm{K}^{+}$efflux independent of P2X7R (51) and the decline of intracellular ATP, of which the latter is considered as a key trigger of NLRP3 inflammasome activation (52) and also another critical condition for TRPM4 channel opening (53). We thus explored the role of SUR1-TRPM4 complex in the P2X7R-independent NLRP3 activation. The nigericin-treated BV2 cells showed increment of caspase-1 activation and mature IL-1 $\beta$ release, but the increase was abolished by 9-Ph or GLB (Fig. 6F-G). Likewise, GLB or 9-Ph abrogated the NLRP3 activation fueled by $\mathrm{NaN}_{3}$ (Fig. $\left.6 \mathrm{H}-\mathrm{I}\right)$, a metabolic toxin depleting intracellular ATP (54), and Fig. $6 \mathrm{~J}$ verified the efficacy of nigericin and $\mathrm{NaN}_{3}$ to reduce intracellular ATP. Overall, these results imply that SUR1-TRPM4 channel participates to the P2X7R-independent NLRP3 inflammasome activation following cytoplasmic ATP decrease in microglia, which may be another NLRP3 activation pathway involving SUR1-TRPM4 in vivo. 


\section{SUR1-TRPM4 magnifies the $\mathrm{K}{ }^{+}$efflux via the $\mathrm{Na}^{+}$influx- driven opening of downstream $\mathrm{K}^{+}$channels in BV2 cells}

Previous studies have illuminated the engagement of multiple specific $\mathrm{K}^{+}$channels belonging to $\mathrm{K}_{2 \mathrm{P}}$ and $\mathrm{Kv}$ family (23-25) in the decline of intracellular $\mathrm{K}^{+}$required for NLRP3 activation. Therefore, we further addressed the $\mathrm{K}^{+}$efflux mechanism involving above channels in the NLRP3 inflammasome activation mediated by SUR1-TRPM4 complex. The results verified that inhibition of Kv1.3 by PAP-1 partially restored the augmented caspase- 1 cleavage and IL-1 $\beta$ release triggered by ATP or diazoxide in LPSprimed BV2 cells, while the NLRP3 activation was almost completely suppressed by quinine, an antagonist of $\mathrm{K}_{2 \mathrm{P}}$ family (Fig. 7A-B). As expected, inhibiting kv1.3 or $\mathrm{K}_{2 \mathrm{p}}$ family counteracted the obvious decline in intracellular $\mathrm{K}^{+}$caused by ATP or diazoxide (Fig. 7C). Moreover, the BV2 cells challenged with LPS manifested the massively increased mRNA levels of representative channels such as TWIK1 (twopore domain weak inwardly rectifying $\mathrm{K}^{+}$channel 1 ), TWIK2, THIK1 (tandem pore domain halothaneinhibited $\mathrm{K}^{+}$channel 1), and Kv1.3 (Fig. 7D). These findings prove that these representative $\mathrm{K}^{+}$channels mediate the $\mathrm{K}^{+}$efflux required for the NLRP3 activation.

Given that the opening of above $\mathrm{K}^{+}$channels was regulated by the depolarized membrane potential (26, 27), we also addressed the mechanism by which SUR1-TRPM4 opened the downstream $\mathrm{K}^{+}$channels. We observed that the caspase- 1 activation and increased supernatant IL-1 $\beta$ was largely reversed, when the LPS-primed BV2 cells treated with ATP or diazoxide were bathed in a nominally $\mathrm{Na}^{+}$-free medium (Fig. 7EF), accompanied by the inhibition of ATP- or diazoxide-induced inward $\mathrm{Na}^{+}$current, depolarized membrane potential, and outward $\mathrm{K}^{+}$current, which was comparable to the efficacy of silencing SUR1TRPM4 (Fig. 7C, 7G-H). These results suggest that SUR1-TRPM4 regulates the $\mathrm{Na}^{+}$influx and subsequent depolarization of membrane potential, thereby opening the mentioned $\mathrm{K}^{+}$channels and amplifying $\mathrm{K}^{+}$ efflux.

\section{NLRP3 inflammasome activation in RAW264.7 cells is attributed to the TRPM4 channel alone rather than binding to SUR1}

Given that $200 \mu \mathrm{mol} / \mathrm{L}$ of GLB but not glipizide entirely inhibits the NLRP3 inflammasome activation in macrophages, suggesting a SUR1-independent inhibitory effect (16), we further explored the difference in the mechanism of NLRP3 activation between microglia and macrophages. As illustrated in Fig. S4A-B, ATP-induced NLRP3 inflammasome activation in RAW264.7 cells challenged with LPS was completely abrogated by $50 \mu \mathrm{mol} / \mathrm{L}$ of 9-Ph, partly restored by $100 \mu \mathrm{mol} / \mathrm{L}$ of GLB, but not recovered by GLZ or GLM. Additionally, TRPM4 was poorly capable of binding to SUR1 in RAW264.7 cells, compared with BV2 cells (Fig. S4C). This was reminiscent of the $\mathrm{K}_{\text {ATP }}$ channel, and we thereby evaluated whether Kir6.x had better 
capacity of competitive binding to SUR1 than TRPM4 in RAW264.7 cells. The levels of SUR1 and TRPM4 were elevated in LPS-treated BV2 and RAW264.7 cells, whereas Kir6.1 level was increased in RAW264.7 but not BV2 cells (Fig. S4D-F). Consistently, SUR1-TRPM4 complex was dominant in BV2 cells, while obvious interaction between SUR1 and Kir6.1 was observed in RAW264.7 cells (Fig. S4G), demonstrating the existence of competitive relationship between TRPM4 and Kir6.1. Therefore, we suppose that the TRPM4 channel mainly exists at an unbound state in macrophages to mediate the NLRP3 inflammasome activation, and can be directly blocked by higher concentration of GLB (50).

\section{Discussion}

In the previous studies, GLB has reportedly played a neuroprotective role in several neurologic diseases such as ischemic stroke (3), subarachnoid hemorrhage (4), and traumatic brain injury (5), likely through blocking the SUR1-TRPM4 complex in neurons and astrocytes, which has been reported to mediate the dramatic influx of $\mathrm{Na}^{+}$and water, strongly correlating with the BBB damage and cerebral edema in the above neurologic diseases (3-5). However, recent studies have also found that the inhibition of SUR1TRPM4 by GLB reduces the neuroinflammatory burden of brain damages without obvious edema (12, 13), implying a complementary neuroprotective mechanism underlying the inhibition of SUR1-TRPM4 with GLB, through restricting neuroinflammation but not reducing edema. NLRP3 inflammasome activation is a critical host defense to danger signals such as extracellular ATP released from dead cells. However, overactivity of this pathway can cause detrimental inflammatory processes and exacerbate tissue damage in various neurological disorders (55-57). In terms of the findings that GLB has been reported to suppress NLRP3 activation in previous studies $(16,58,59)$, we speculate that GLB prevents the activation of NLRP3 inflammasome via blocking SUR1-TRPM4.

In recent years, our team has observed that SUR1-TRPM4 is highly expressed in murine microglia after $\mathrm{CA} / \mathrm{CPR}$ or ischemic stroke $(47,60)$. Moreover, the critical role of microglia in providing the platform for neuroinflammation is further exemplified by our and others' findings that the aberrant NLRP3 inflammasome activation in microglia mediates the excessive neuroinflammation in CA/CPR and RBI models $(17,30)$. In view of the finding that the expression of certain pro-inflammatory genes could be downregulated via blocking the upregulated SUR1-TRPM4 channel with GLB in LPS-activated microglia (12), we further hypothesize that GLB effectively prevents microglial NLRP3 activation through inhibiting SUR1-TRPM4. Here, we conducted in vivo experiments in the CA/CPR and RBI models, and the two models were mainly characterized by neuroinflammation. As indicated by our evidence, almost no brain edema was observed in the RBI model, thus minimizing the interference of reducing edema with GLB. We demonstrated that the microglial NLRP3 activation after CA/CPR or whole-brain radiation could be abolished by GLB. The most important point was that treatment with GLB in irradiated Trpm $4^{-1-}$ mice showed no better performance in abrogating NLRP3 activation, compared to the irradiated Trpm4 $4^{-/-}$mice without GLB, suggesting that SUR1-TRPM4 indeed acts as the specific target of GLB. Subsequently, BV2 cells were treated with LPS, radiation, and OGD/R to mimic the pathophysiologic process in CA/CPR and RBI models, further indicating that the sequential activation of SUR1-TRPM4 and NLRP3 inflammasome

Page $18 / 36$ 
acts as common response to damage stress in microglia challenged with various stimuli, directly participates in the injurious neuroinflammation, and can be prevented by GLB through occluding SUR1TRPM4. Besides, we confirmed that SUR1-TRPM4 also participated in the P2X7R-independent NLRP3 activation initiated by reduced cytoplasmic ATP in microglia, and could also be inhibited by GLB. It was noteworthy that the intracellular ATP was partially decreased in OGD/R microglia, implying that SUR1TRPM4 may mediate the NLRP3 activation via two diverse routes simultaneously after OGD/R. Therefore, it may be a complementary route of SUR1-TRPM4-involved NLRP3 activation in vivo. Then we further addressed whether the anti-inflammatory effects of GLB exerted complementary neuroprotection in inflammatory brain damages after CA/CPR or whole-brain radiation. Inspiringly, the results showed that GLB could significantly improve neurologic outcomes, promote the recovery of cognitive function, and alleviate the neuropathologic injuries in CA/CPR and RBI models, strongly implying that GLB has beneficial effects on neurologic diseases via reducing not only edema but also inflammation.

Microglial functions are closely related to ion channels (61). Previous study has proved that the reduction of intracellular $\mathrm{K}^{+}$to $70 \%-80 \%$ is sufficient to activate NLRP3 (22), suggesting the critical role of $\mathrm{K}^{+}$ channels in NLRP3 inflammasome activation. P2X7R is a non-selective cation channel which acts as an initial trigger of NLRP3 activation by sensing extracellular ATP (20), but the $\mathrm{K}^{+}$outward current required for NLRP3 activation is mainly mediated by other $\mathrm{K}^{+}$channels like $\mathrm{K}_{2 \mathrm{P}}$ family and $\mathrm{Kv} 1.3$ (23-25). Likewise, we observed that the mRNA levels of TWIK1, TWIK2, THIK1, and Kv1.3 were obviously upregulated in LPS-primed microglia, and the above channels collaboratively launched the ATP-induced NLRP3 activation, of which $\mathrm{K}_{2 \mathrm{P}}$ family played a dominant role, as reflected by the result that quinine almost entirely counteracted the NLRP3 activation, consistent with previous studies $(23,24)$. It is reported that THIK-1 is not expressed in cultured microglia (62), conflicting with our result of highly expressed THIK-1 after LPS treatment, which may be attributed to the non-modeling microglia used in the previous study, with different cell status from ours. However, the underlying mechanism by which involved channel-mediated $\mathrm{K}^{+}$efflux is triggered after activating P2X7R remains controversial. In vitro, we found that SUR1-TRPM4 could be fueled through sensing increased intracellular $\mathrm{Ca}^{2+}$ mediated by P2X7R, and gave rise to the $\mathrm{Na}^{+}$inward current responsible for the onset of $\mathrm{K}^{+}$efflux. In summary, we first illustrate that SUR1-TRPM4 acts as a signal amplifier and an integrated switch of downstream $\mathrm{K}^{+}$channels, and ultimately triggers $\mathrm{K}^{+}$efflux-driven NLRP3 activation, through mediating $\mathrm{Na}^{+}$influx and consequential depolarized membrane potential (Fig. 8). The opening mechanisms of related $\mathrm{K}^{+}$channels: 1) $\mathrm{K}_{2 \mathrm{P}}$ family members belong to the constitutively active $\mathrm{K}^{+}$leak channels, and the depolarized membrane potential magnifies the driving force of $\mathrm{K}^{+}$efflux (27); 2) Kv1.3 belongs to voltage-gated $\mathrm{K}^{+}$channels, and is activated by the depolarized membrane potential (26).

High-dose GLB $(200 \mu \mathrm{mol} / \mathrm{L})$ can reverse the NLRP3 activation in macrophages (16), but there are major challenges towards clinical translation due to the side effect of hypoglycemia. In this study, we found that NLRP3 inflammasome activation in microglia could be entirely suppressed by $100 \mu \mathrm{mol} / \mathrm{L}$ of sulfonylureas including GLB. Therefore, we further provided important insights into the differences in the 
mechanism under NLRP3 activation between microglia and macrophages. In view of another finding that SUR1 tended to bind TRPM4 in microglia, but Kir6.1 in macrophages, we support the notion that TRPM4 in macrophages mainly participates in the NLRP3 activation at an unbound state, and is directly blocked by higher dose of GLB because of lower sensitivity to GLB compared to SUR1-TRPM4 $(12,50)$, whereas SUR1-TRPM4 in microglia can be inhibited by both GLB and other sulfonylureas. On account of the minimal risk of hypoglycemia (63), we hope that our research will represent a turning point in the understanding of the direct anti-inflammatory effects of GLB, and promote the translation of low-dose GLB into clinical trials for neurological disorders involving NLRP3-related inflammation.

\section{Conclusion}

This study uncovers a complementary neuroprotective mechanism of GLB, which is attributed to the inhibition of NLRP3-mediated neuroinflammation through blocking SUR1-TRPM4 in microglia, and independent of the well-documented neuroprotection through blocking SUR1-TRPM4 in neurovascular units and thus reducing the brain edema. Mechanistically, this research reveals that the SUR1-TRPM4 complex serves as a $\mathrm{K}^{+}$efflux amplifier in activating the NLRP3 inflammasome in microglia, via mediating $\mathrm{Na}^{+}$influx, contributing to the depolarized membrane potential, and thereby gating downstream $\mathrm{K}^{+}$channels. In conclusion, this study will certainly be insightful for deciphering the direct anti-inflammatory effects of GLB, as well as the contribution of low-dose GLB to combating neurological disorders involving NLRP3-related inflammation.

\section{Abbreviations}

ATP: adenosine triphosphate; BBB: blood-brain barrier; BBG: Brilliant Blue G; CA/CPR: cardiac arrest/cardiopulmonary resuscitation; DAMPs: danger-associated molecular patterns; DCX: Doublecortin; DMSO: dimethyl sulfoxide; FBS: fetal bovine serum; GFAP: glial fibrillary acidic protein; GLB: Glibenclamide; GLM: glimepiride; GLZ: gliclazide; Iba-1: ionized calcium-binding adapter molecule-1; IL$1 \beta$ : interleukin-1 $\beta$; $\mathrm{K}_{2 \mathrm{P}}$ family: two-pore domain $\mathrm{K}^{+}$channel family; $\mathrm{Kv}$ family: voltage-gated $\mathrm{K}^{+}$channel family; LPS: lipopolysaccharides; MAP: mean arterial pressure; MAP-2: microtubule-associated protein 2; NDSs: neurologic deficit scores; NeuN: neuronal nuclei; NF-KB: nuclear factor kappa-B; NLRP3: nucleotidebinding oligomerization domain-like receptor containing pyrin domain 3; OGD/R: oxygen-glucose deprivation/reperfusion; PFA: paraformaldehyde; pro-caspase-1: precursor of caspase-1; pro-IL1 $\beta$ : precursor of IL-1 $\beta$; P2X7R: P2X7 receptor; qRT-PCR: quantitative real-time polymerase chain reaction; RBI: Radiation-Induced Brain Injury; ROSC: return of spontaneous circulation; Sp-1: specificity protein 1; SUR1-TRPM4: Sulfonylurea receptor 1-transient receptor potential M4; THIK1: tandem pore domain halothane-inhibited $\mathrm{K}^{+}$channel 1; TLR: Toll-like receptor; TWIK1: two-pore domain weak inwardly rectifying $\mathrm{K}^{+}$channel 1; 9-Ph: 9-phenanthrol.

\section{Declarations}


Acknowledgments

We thank the central laboratory of Southern Medical University for technical assistance in rodent MRI scanning and brain radiation modeling.

\section{Author Contributions}

Y.H. and Y.C. performed all the experiments and wrote the manuscript. Y.P., J.Z., K.L., J.C., Y.W., Z.J., Z.L., S.W., S.G. and N.Z. completed the statistical analysis. S.P. and K.H. designed, guided the experiments and critically revised the manuscript.

\section{Funding}

This work was supported by the National Key R\&D Program of China (No. 2017YFC1307500), National Natural Science Foundation of China (No. 81701294 \& 82072133 \& 81871030), and Guangdong Basic and Applied Basic Research Foundation (2019A1515011446 \& 2021A1515010922).

\section{Availability of data and materials}

The datasets used and/or analyzed during this study are available from the corresponding authors on reasonable request.

\section{Ethics approval and consent to participate}

All animal experiments in this study were approved by Animal Care and Use Committee of Nanfang Hospital, Southern Medical University (Guangzhou, China).

\section{Consent for publication}

Not applicable.

\section{Competing Interests}

The authors declare that they have no competing interests.

\section{Author details}

${ }^{1}$ Department of Neurology, Nanfang Hospital, Southern Medical Univerisity, Guangzhou North Avenue 1838\#, Guangzhou 510515, China.

\section{References}

1. J. M. Simard, S. K. Woo, S. Bhatta, V. Gerzanich, Drugs acting on SUR1 to treat CNS ischemia and trauma. Curr. Opin. Pharmacol. 8, 42-49 (2008). 
2. J. Guo, J. She, W. Zeng, Q. Chen, X. Bai, Y. Jiang, Structures of the calcium-activated, non-selective cation channel TRPM4. Nature 552, 205-209 (2017).

3. J. M. Simard, M. Chen, K. V. Tarasov, S. Bhatta, S. Ivanova, L. Melnitchenko, N. Tsymbalyuk, G. A. West, V. Gerzanich, Newly expressed SUR1-regulated NCCa-ATP channel mediates cerebral edema after ischemic stroke. Nat. Med. 12, 433-440 (2006).

4. J. M. Simard, Z. Geng, S. K. Woo, S. Ivanova, C. Tosun, L. Melnichenko, V. Gerzanich, Glibenclamide reduces inflammation, vasogenic edema, and caspase-3 activation after subarachnoid hemorrhage. J. Cereb. Blood Flow Metab. 29, 317-330 (2009).

5. K. Zweckberger, K. Hackenberg, C. S. Jung, D. N. Hertle, K. L. Kiening, A. W. Unterberg, O. W. Sakowitz, Glibenclamide reduces secondary brain damage after experimental traumatic brain injury. Neuroscience 272, 199-206 (2014).

6. J. M. Simard, O. Tsymbalyuk, A. Ivanov, S. Ivanova, S. Bhatta, Z. Geng, S. K. Woo, V. Gerzanich, Endothelial sulfonylurea receptor 1-regulated NCCa-ATP channels mediate progressive hemorrhagic necrosis following spinal cord injury. J. Clin. Invest. 117, 2105-2113 (2007).

7. W. T. Kimberly, M. B. Bevers, R. von Kummer, A. M. Demchuk, J. M. Romero, J. J. Elm, H. E. Hinson, B. J. Molyneaux, J. M. Simard, K. N. Sheth, Effect of IV glyburide on adjudicated edema endpoints in the GAMES-RP trial. Neurology 91, e2163-e2169 (2018).

8. K. N. Sheth, N. H. Petersen, K. Cheung, J. J. Elm, H. E. Hinson, B. J. Molyneaux, L. A. Beslow, G. K. Sze, J. M. Simard, W. T. Kimberly, Long-term outcomes in patients aged $\leq 70$ years with intravenous glyburide from the phase II GAMES-RP study of large hemispheric infarction: an exploratory analysis. Stroke 49, 1457-1463 (2018).

9. K. Huang, Y. Hu, Y. Wu, Z. Ji, S. Wang, Z. Lin, J. Xu, S. Pan, Exploratory analysis of oral glibenclamide in acute ischemic stroke. Acta Neurol. Scand. 140, 212-218 (2019).

10. H. Kunte, M. A. Busch, K. Trostdorf, B. Vollnberg, L. Harms, R. I. Mehta, R. J. Castellani, P. Mandava, T. A. Kent, J. M. Simard, Hemorrhagic transformation of ischemic stroke in diabetics on sulfonylureas. Ann. Neurol. 72, 799-806 (2012).

11. J. J. Chang, Y. Khorchid, A. Kerro, L. G. Burgess, N. Goyal, A. W. Alexandrov, A. V. Alexandrov, G. Tsivgoulis, Sulfonylurea drug pretreatment and functional outcome in diabetic patients with acute intracerebral hemorrhage. J. Neurol. Sci. 381, 182-187 (2017).

12. D. B. Kurland, V. Gerzanich, J. K. Karimy, S. K. Woo, R. Vennekens, M. Freichel, B. Nilius, J. Bryan, J. M. Simard, The Sur1-Trpm4 channel regulates NOS2 transcription in TLR4-activated microglia. J. Neuroinflammation 13, 130 (2016).

13. T. K. Makar, V. Gerzanich, V. K. C. Nimmagadda, R. Jain, K. Lam, F. Mubariz, D. Trisler, S. Ivanova, S. K. Woo, M. S. Kwon, J. Bryan, C. T. Bever, J. M. Simard, Silencing of Abcc8 or inhibition of newly upregulated Sur1-Trpm4 reduce inflammation and disease progression in experimental autoimmune encephalomyelitis. J. Neuroinflammation 12, 210 (2015).

14. E. Latz, T. S. Xiao, A. Stutz, Activation and regulation of the inflammasomes. Nat. Rev. Immunol. 13, 397-411 (2013). 
15. M. Lamkanfi, V. M. Dixit, Inflammasomes: guardians of cytosolic sanctity. Immunol. Rev. 227, 95-105 (2009).

16. M. Lamkanfi, J. L. Mueller, A. C. Vitari, S. Misaghi, A. Fedorova, K. Deshayes, W. P. Lee, H. M. Hoffman, V. M. Dixit, Glyburide inhibits the Cryopyrin/Nalp3 inflammasome. J. Cell Biol. 187, 61-70 (2009).

17. Y. Chang, J. Zhu, D. Wang, H. Li, Y. He, K. Liu, X. Wang, Y. Peng, S. Pan, K. Huang, NLRP3 inflammasome-mediated microglial pyroptosis is critically involved in the development of postcardiac arrest brain injury. J. Neuroinflammation 17, 219 (2020).

18. H. Guo, J. B. Callaway, J. P. Ting, Inflammasomes: mechanism of action, role in disease, and therapeutics. Nat. Med. 21, 677-687 (2015).

19. A. Surprenant, F. Rassendren, E. Kawashima, R. A. North, G. Buell, The cytolytic $P_{2 Z}$ receptor for

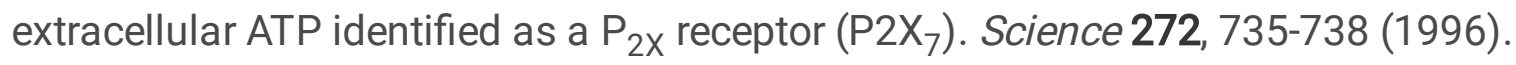

20. F. Di Virgilio, D. Dal Ben, A. C. Sarti, A. L. Giuliani, S. Falzoni, The P2X7 receptor in infection and inflammation. Immunity 47, 15-31 (2017).

21. G. Y. Chen, G. Nuñez, Sterile inflammation: sensing and reacting to damage. Nat. Rev. Immunol. 10, 826-837 (2010).

22. R. Muñoz-Planillo, P. Kuffa, G. Martínez-Colón, B. L. Smith, T. M. Rajendiran, G. Núñez, $K^{+}$efflux is the common trigger of NLRP3 inflammasome activation by bacterial toxins and particulate matter. Immunity 38, 1142-1153 (2013).

23. A. Di, S. Xiong, Z. Ye, R. K. S. Malireddi, S. Kometani, M. Zhong, M. Mittal, Z. Hong, T. Kanneganti, J. Rehman, A. B. Malik, The TWIK2 potassium efflux channel in macrophages mediates NLRP3 inflammasome-induced inflammation. Immunity 49, 56-65 (2018).

24. C. Madry, V. Kyrargyri, I. L. Arancibia-Cárcamo, R. Jolivet, S. Kohsaka, R. M. Bryan, D. Attwell, Microglial ramification, surveillance, and interleukin-1 $\beta$ release are regulated by the two-pore domain $\mathrm{K}^{+}$channel THIK-1. Neuron 97, 299-312 (2018).

25. D. Ma, N. Zhang, Y. Zhang, H. Chen, Kv1.3 channel blockade alleviates cerebral ischemia/reperfusion injury by reshaping M1/M2 phenotypes and compromising the activation of NLRP3 inflammasome in microglia. Exp. Neurol. 332, 113399 (2020).

26. H. Wulff, B. S. Zhorov, $\mathrm{K}^{+}$channel modulators for the treatment of neurological disorders and autoimmune diseases. Chem. Rev. 108, 1744-1773 (2008).

27. P. Enyedi, G. Czirják, Molecular background of leak $\mathrm{K}^{+}$currents: two-pore domain potassium channels. Physiol. Rev. 90, 559-605 (2010).

28. K. Huang, Y. Gu, Y. Hu, Z. Ji, S. Wang, Z. Lin, X. Li, Z. Xie, S. Pan, Glibenclamide improves survival and neurologic outcome after cardiac arrest in rats. Crit. Care Med. 43, e341-e349 (2015).

29. Z. Lin, H. Huang, Y. Gu, K. Huang, Y. Hu, Z. Ji, Y. Wu, S. Wang, T. Yang, S. Pan, Glibenclamide ameliorates cerebral edema and improves outcomes in a rat model of status epilepticus. Neuropharmacology 121, 1-11 (2017). 
30. Y. Peng, K. Lu, Z. Li, Y. Zhao, Y. Wang, B. Hu, P. Xu, X. Shi, B. Zhou, M. Pennington, K. G. Chandy, Y. Tang, Blockade of Kv1.3 channels ameliorates radiation-induced brain injury. Neuro. Oncol. 16, 528539 (2014).

31. B. Schattling, K. Steinbach, E. Thies, M. Kruse, A. Menigoz, F. Ufer, V. Flockerzi, W. Brück, O. Pongs, R. Vennekens, M. Kneussel, M. Freichel, D. Merkler, M. A. Friese, TRPM4 cation channel mediates axonal and neuronal degeneration in experimental autoimmune encephalomyelitis and multiple sclerosis. Nat. Med. 18, 1805-1811 (2012).

32. S. Hwang, J. Jung, T. Kim, S. Lim, E. Oh, J. Kim, K. Ji, E. Joe, K. Cho, I. Han, Ionizing radiation induces astrocyte gliosis through microglia activation. Neurobiol. Dis. 21, 457-467 (2006).

33. Y. Ye, T. Jin, X. Zhang, Z. Zeng, B. Ye, J. Wang, Y. Zhong, X. Xiong, L. Gu, Meisoindigo protects against focal cerebral ischemia-reperfusion injury by inhibiting NLRP3 inflammasome activation and regulating microglia/macrophage polarization via TLR4/NF-kB signaling pathway. Front. Cell Neurosci. 13, 553 (2019).

34. S. Zhu, J. Huang, W. Peng, D. Wu, M. Luo, Q. Li, Z. Li, X. Feng, F. Liu, M. Wang, W. Chen, N. Olsen, S. Zheng, Inhibition of smoothened decreases proliferation of synoviocytes in rheumatoid arthritis. Cell Mol. Immunol. 14, 214-222 (2017).

35. H. Ding, H. Zhang, H. Ding, D. Li, X. Yi, X. Ma, R. Li, M. Huang, X. Ju, Transplantation of placentaderived mesenchymal stem cells reduces hypoxic-ischemic brain damage in rats by ameliorating the inflammatory response. Cell Mol. Immunol. 14, 693-701 (2017).

36. R. G. Geocadin, R. Ghodadra, T. Kimura, H. Lei, D. L. Sherman, D. F. Hanley, N. V. Thakor, A novel quantitative EEG injury measure of global cerebral ischemia. Clin. Neurophysiol. 111, 1779-1787 (2000).

37. D. Luo, W. Ge, X. Hu, C. Li, C. Lee, L. Zhou, Z. Wu, J. Yu, S. Lin, J. Yu, W. Xu, L. Chen, C. Zhang, K. Jiang, X. Zhu, H. Li, X. Gao, Y. Geng, B. Jing, Z. Wang, C. Zheng, R. Zhu, Q. Yan, Q. Lin, K. Ye, Y. Sun, L. Cheng, Unbiased transcriptomic analyses reveal distinct effects of immune deficiency in CNS function with and without injury. Protein Cell 10, 566-582 (2019).

38. S. L. Lage, V. M. Dominical, C. Wong, I. Sereti, Evaluation of canonical inflammasome activation in human monocytes by imaging flow cytometry. Front. Immunol. 10, 1284 (2019).

39. S. Hassan, K. Eldeeb, P. J. Millns, A. J. Bennett, S. P. H. Alexander, D. A. Kendall, Cannabidiol enhances microglial phagocytosis via transient receptor potential (TRP) channel activation. Br. J. Pharmacol. 171, 2426-2439 (2014).

40. B. Seligmann, J. I. Gallin, Secretagogue modulation of the response of human neutrophils to chemoattractants: studies with a membrane potential sensitive cyanine dye. Mol. Immunol. 17, 191200 (1980).

41. Y. Tao, D. Yan, Q. Yang, R. Zeng, Y. Wang, Low K+ promotes NF-kappaB/DNA binding in neuronal apoptosis induced by K+ loss. Mol. Cell Biol. 26, 1038-1050 (2006).

42. B. Yang, L. Zhang, Y. Cao, S. Chen, J. Cao, D. Wu, J. Chen, H. Xiong, Z. Pan, F. Qiu, J. Chen, X. Ling, M. Yan, S. Huang, S. Zhou, T. Li, L. Yang, Y. Huang, J. Lu, Overexpression of IncRNA IGFBP4-1 
reprograms energy metabolism to promote lung cancer progression. Mol. Cancer 16, 154 (2017).

43. H. Liao, H. Wang, X. Rong, E. Li, R. H. Xu, Y. Peng, Mesenchymal stem cells attenuate radiationinduced brain injury by inhibiting microglia pyroptosis. Biomed. Res. Int. 2017, 1948985 (2017).

44. M. T. Makale, C. R. McDonald, J. A. Hattangadi-Gluth, S. Kesari, Mechanisms of radiotherapyassociated cognitive disability in patients with brain tumours. Nat. Rev. Neurol. 13, 52-64 (2017).

45. M. L. Monje, S. Mizumatsu, J. R. Fike, T. D. Palmer, Irradiation induces neural precursor-cell dysfunction. Nat. Med. 8, 955-962 (2002).

46. K. Huang, Z. Wang, Y. Gu, Z. Ji, Z. Lin, S. Wang, S. Pan, Y. Wu, Glibenclamide prevents water diffusion abnormality in the brain after cardiac arrest in rats. Neurocrit. Care 29, 128-135 (2018).

47. K. Huang, Z. Wang, Y. Gu, Y. Hu, Z. Ji, S. Wang, Z. Lin, X. Li, Z. Xie, S. Pan, Glibenclamide is comparable to target temperature management in improving survival and neurological outcome after asphyxial cardiac arrest in rats. J. Am. Heart Assoc. 5, e003465 (2016).

48. K. V. Swanson, M. Deng, J. P. Ting, The NLRP3 inflammasome: molecular activation and regulation to therapeutics. Nat. Rev. Immunol. 19, 477-489 (2019).

49. S. Ming, L. Zeng, Y. Guo, S. Zhang, G. Li, Y. Ma, Y. Zhai, W. Chang, L. Yang, J. Wang, G. Yang, B. Chu, The human-specific STING agonist G10 activates type I interferon and the NLRP3 inflammasome in porcine cells. Front. Immunol. 11, 575818 (2020).

50. S. K. Woo, M. S. Kwon, A. Ivanov, V. Gerzanich, J. M. Simard, The sulfonylurea receptor 1 (Sur1)transient receptor potential melastatin 4 (Trpm4) channel. J. Biol. Chem. 288, 3655-3667 (2013).

51. M. A. Katsnelson, L. G. Rucker, H. M. Russo, G. R. Dubyak, $K^{+}$efflux agonists induce NLRP3 inflammasome activation independently of $\mathrm{Ca}^{2+}$ signaling. J. Immunol. 194, 3937-3952 (2015).

52. J. Nomura, A. So, M. Tamura, N. Busso, Intracellular ATP decrease mediates NLRP3 inflammasome activation upon nigericin and crystal stimulation. J. Immunol. 195, 5718-5724 (2015).

53. B. Nilius, J. Prenen, T. Voets, G. Droogmans, Intracellular nucleotides and polyamines inhibit the $\mathrm{Ca}^{2+}$ activated cation channel TRPM4b. Pflugers Arch. 448, 70-75 (2004).

54. M. Chen, J. M. Simard, Cell swelling and a nonselective cation channel regulated by internal $\mathrm{Ca}^{2+}$ and ATP in native reactive astrocytes from adult rat brain. J. Neurosci. 21, 6512-6521 (2001).

55. H. Zhao, Y. Chen, H. Feng, P2X7 receptor-associated programmed cell death in the pathophysiology of hemorrhagic stroke. Curr. Neuropharmacol. 16, 1282-1295 (2018).

56. S. W. Lee, J. P. de Rivero Vaccari, J. S. Truettner, W. D. Dietrich, R. W. Keane, The role of microglial inflammasome activation in pyroptotic cell death following penetrating traumatic brain injury. J. Neuroinflammation 16, 27 (2019).

57. P. Xu, X. Zhang, Q. Liu, Y. Xie, X. Shi, J. Chen, Y. Li, H. Guo, R. Sun, Y. Hong, X. Liu, G. Xu, Microglial TREM-1 receptor mediates neuroinflammatory injury via interaction with SYK in experimental ischemic stroke. Cell Death Dis. 10, 555 (2019).

58. N. A. Hirshman, F. J. Hughes, H. Jin, W. T. Harrison, S. W. White, I. Doan, S. N. Harper, P. D. Leidig, J. T. Purves, Cyclophosphamide-induced cystitis results in NLRP3-mediated inflammation in the 
hippocampus and symptoms of depression in rats. Am. J. Physiol. Renal Physiol. 318, F354-F362 (2020).

59. L. Hou, J. Yang, S. Li, R. Huang, D. Zhang, J. Zhao, Q. Wang, Glibenclamide attenuates 2,5hexanedione-induced neurotoxicity in the spinal cord of rats through mitigation of NLRP3 inflammasome activation, neuroinflammation and oxidative stress. Toxicol. Lett. 331, 152-158 (2020).

60. X. Wang, Y. Chang, Y. He, C. Lyu, H. Li, J. Zhu, K. Liu, Y. Hu, K. Huang, S. Pan, Glimepiride and glibenclamide have comparable efficacy in treating acute ischemic stroke in mice. Neuropharmacology 162, 107845 (2020).

61. P. Izquierdo, D. Attwell, C. Madry, lon channels and receptors as determinants of microglial function. Trends Neurosci. 42, 278-292 (2019).

62. O. Butovsky, M. P. Jedrychowski, C. S. Moore, R. Cialic, A. J. Lanser, G. Gabriely, T. Koeglsperger, B. Dake, P. M. Wu, C. E. Doykan, Z. Fanek, L. Liu, Z. Chen, J. D. Rothstein, R. M. Ransohoff, S. P. Gygi, J. P. Antel, H. L. Weiner, Identification of a unique TGF- $\beta$-dependent molecular and functional signature in microglia. Nat. Neurosci. 17, 131-143 (2014).

63. J. M. Simard, V. Yurovsky, N. Tsymbalyuk, L. Melnichenko, S. Ivanova, V. Gerzanich, Protective effect of delayed treatment with low-dose glibenclamide in three models of ischemic stroke. Stroke $\mathbf{4 0 , 6 0 4 -}$ 609 (2009).

\section{Figures}


A

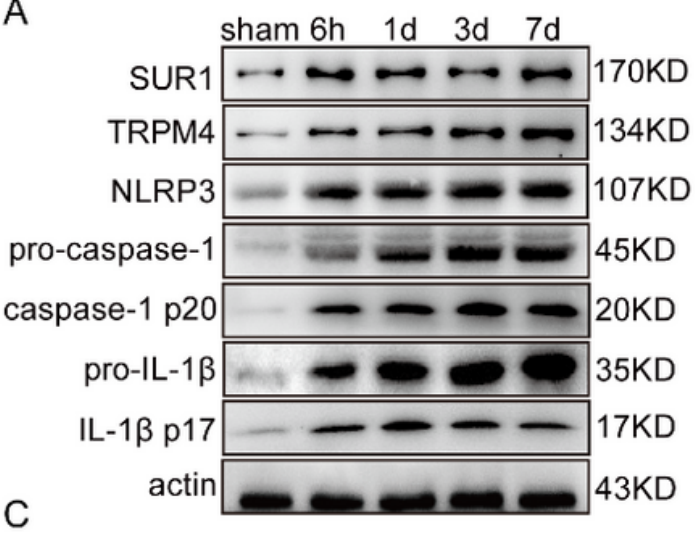

SUR1/TRPM4/DAPI
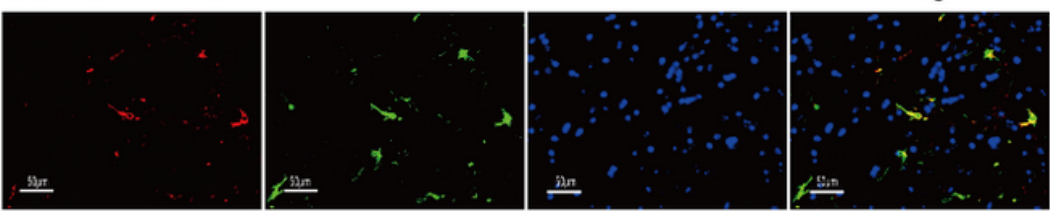

CD68/TRPM4/DAPI
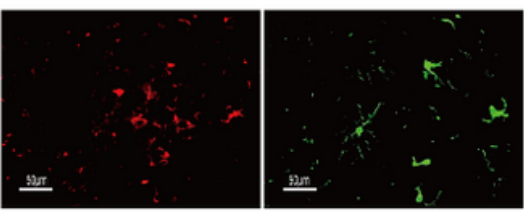

B

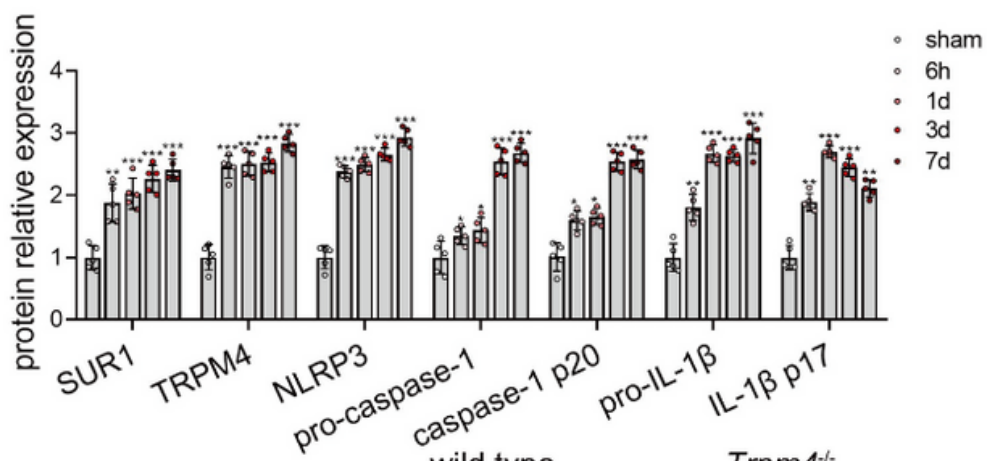

wild type

Trpm4

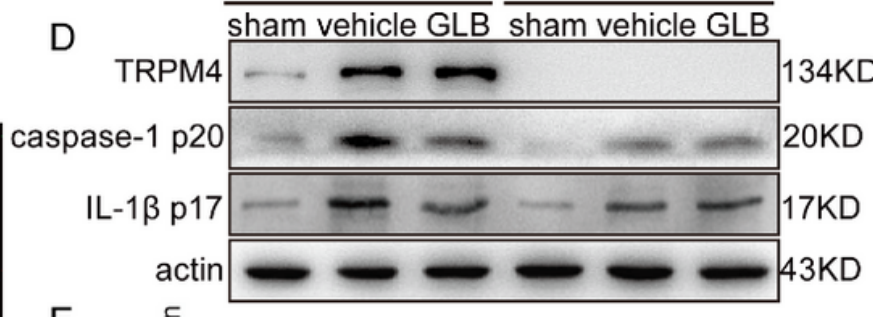

E

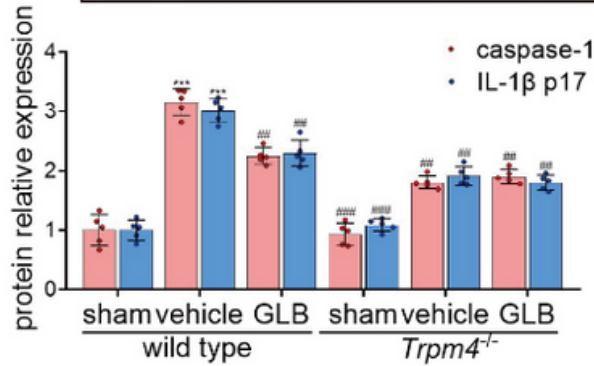

\section{Figure 1}

Inhibition of SUR1-TRPM4 with GLB suppresses NLRP3 activation in microglia after brain radiation. (A and B) Western blotting results of SUR1, TRPM4, and NLRP3 inflammasome-related molecules in shamoperated and irradiated mice that were euthanized at different time points after brain radiation. (C) Confocal analysis of TRPM4 (green) and SUR1 (red) or CD68 (red) staining 7 days after brain radiation. Scale bar $=50 \mu \mathrm{m}$. (D and E) Western blotting results of TRPM4, caspase-1 p20, and IL-1 $\beta$ p17 from wild type or Trpm4-/- mice on day 7 after undergoing different treatments. 6 h, 6 hours. 1 d, 1 day. 3d, 3 days. 7d, 7 days. Statistical significances are determined with one-way ANOVA followed by Tukey's post hoc test. Data are represented as mean $\pm S D$. ${ }^{*} P<0.05,{ }^{*} P<0.01, * \star * P<0.001$ versus wild type sham; \#P $<0.05$, \#\#P <0.01, \#\#\# $\mathrm{P}<0.001$ versus wild type vehicle. $\mathrm{n}=5$ per group. 

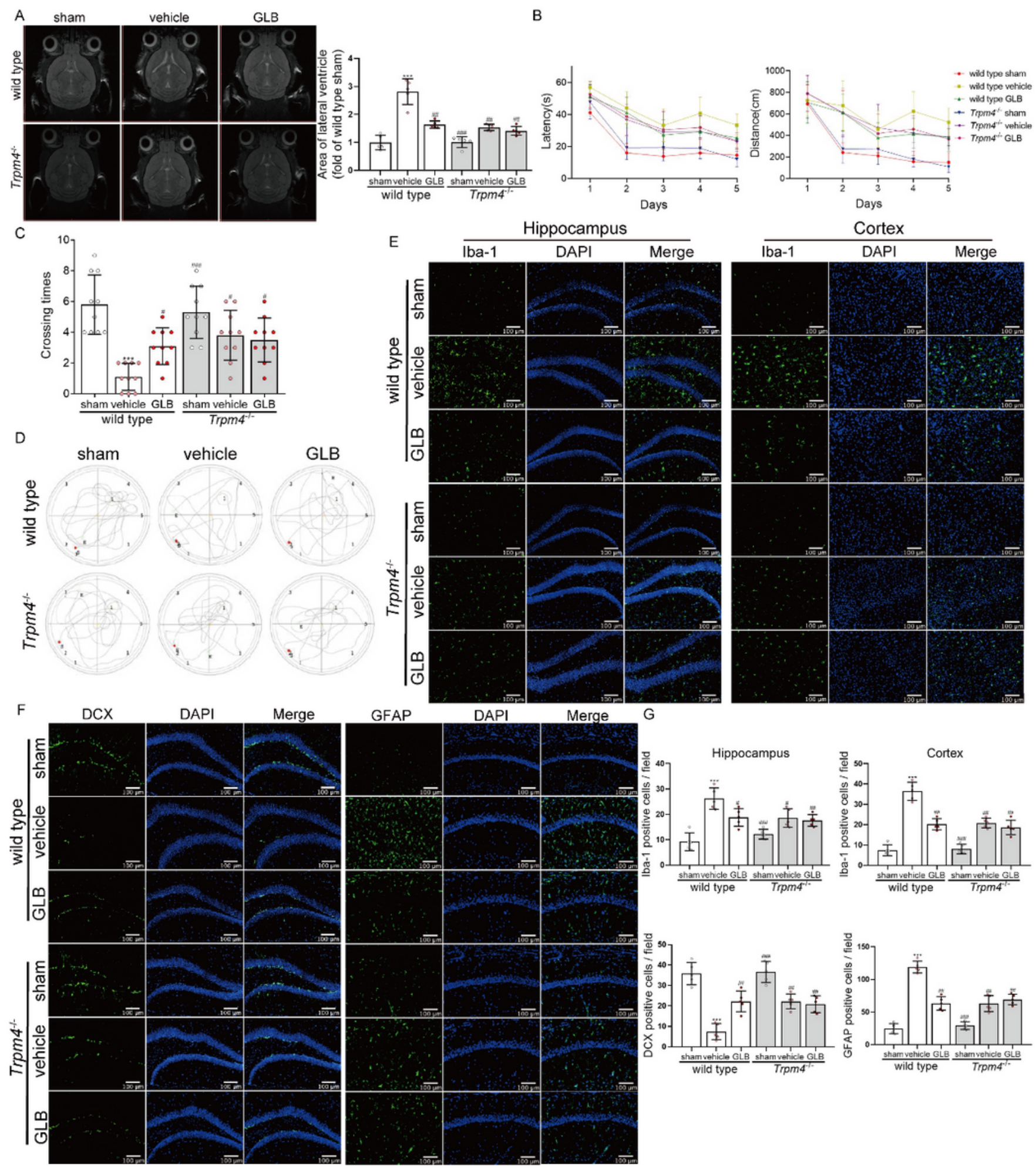

\section{Figure 2}

Inhibiting SUR1-TRPM4 with GLB ameliorates brain atrophy, cognitive impairment and neuropathological damage in RBI model. (A) The MRI scanning results to evaluate the severity of brain atrophy and the area of lateral ventricles of indicated groups 8 weeks post-radiation. (B-D) The effect of blocking SUR1-TRPM4 on post-brain radiation spatial memory and learning deficits assessed by Morris Water Maze analysis 8 weeks post-radiation, including the mean latency and distance to reach the hiding platform (B), the 
frequency of crossing the platform area (C) during the probe trial (D). (E-G) The effect of blocking SUR1TRPM4 on post-brain radiation neuropathological damage characterized by the changes in immunofluorescent staining for Iba-1 in hippocampal DG region and cortex (E), DCX in hippocampal DG region, and GFAP in hippocampus $(\mathrm{F})$. Scale bar $=100 \mu \mathrm{m}$. All data in this figure are analyzed using oneway ANOVA followed by Tukey's post hoc test, except the data of escape latency and distance which are analyzed with repeated-measures ANOVA. Data are represented as mean $\pm S D$. ${ }^{*} P<0.05,{ }^{*} \mathrm{P}<0.01, \star \star \star P<$ 0.001 versus wild type sham; \#P $<0.05$, \#\#P $<0.01$, \#\#\# $P<0.001$ versus wild type vehicle. $n=5$ or 10 per group. 
A

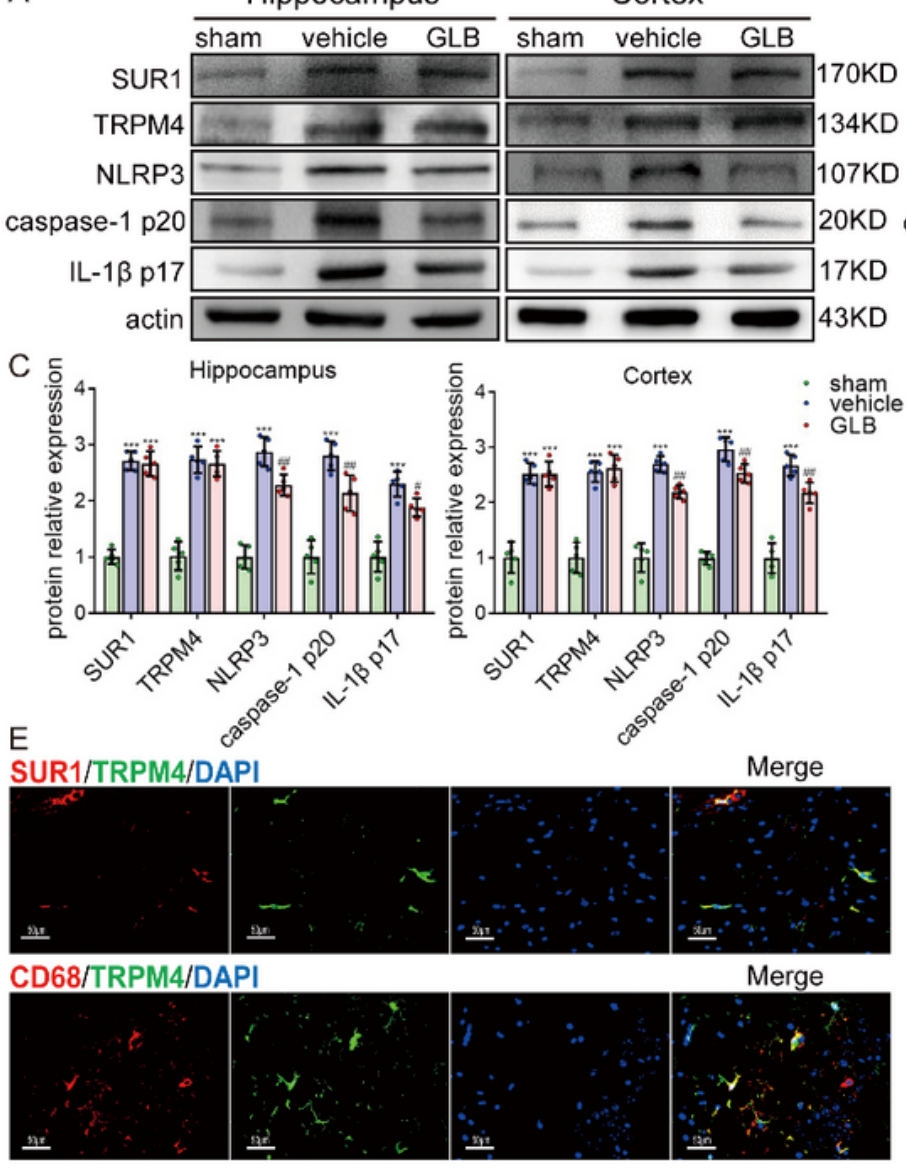

$\mathrm{H}$
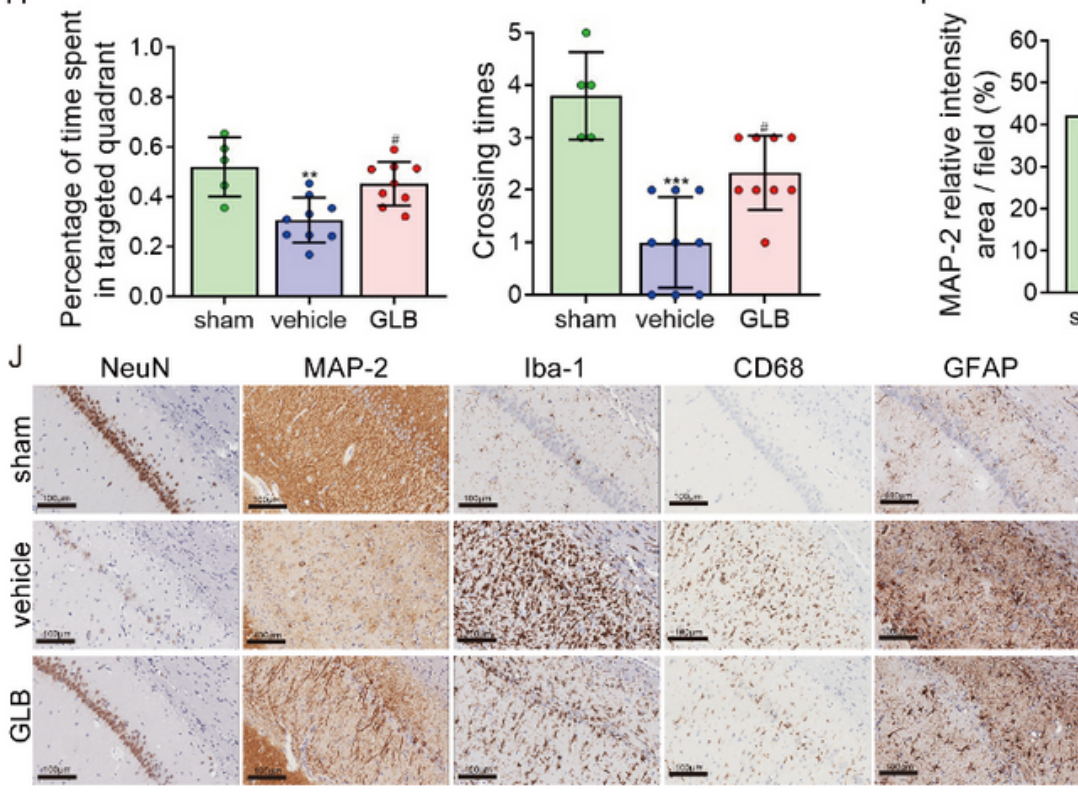

B
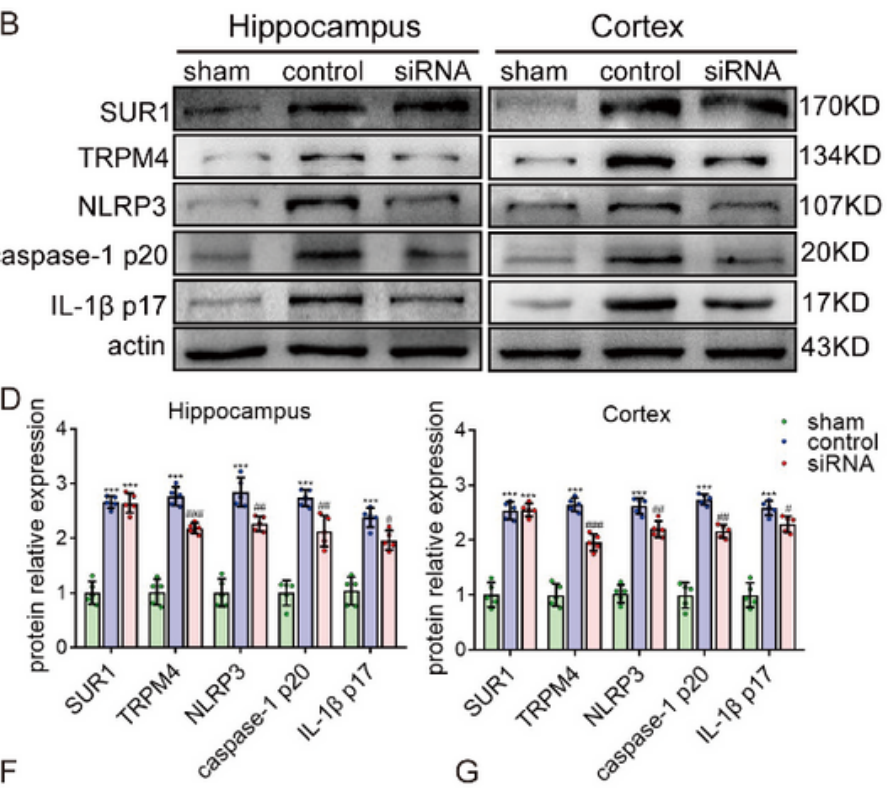

F
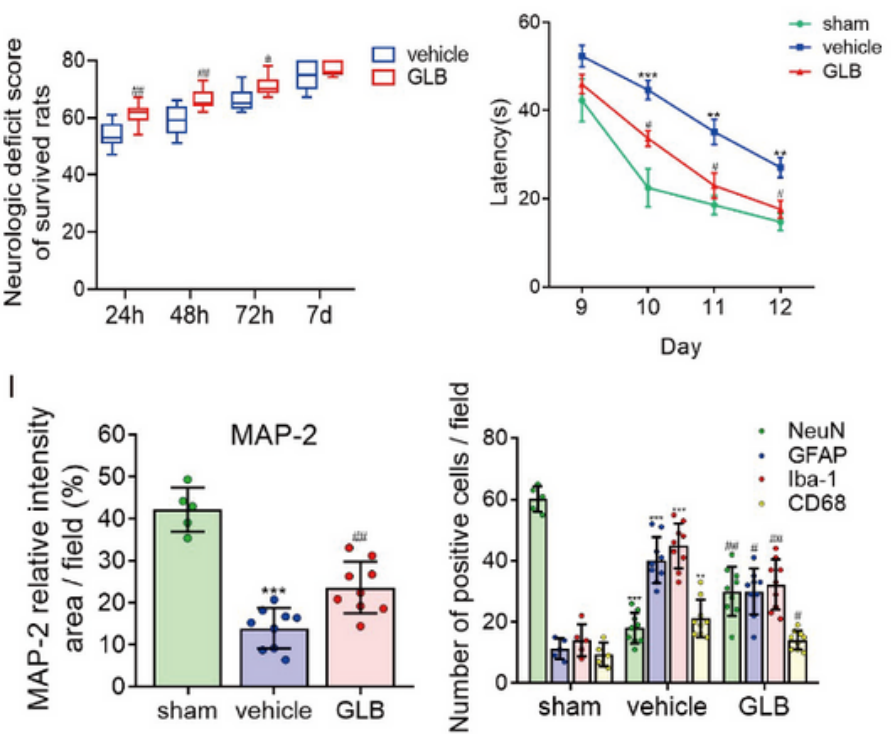

\section{Figure 3}

Blocking SUR1-TRPM4 with GLB reverses NLRP3 inflammasome activation in microglia, and improves neurocognitive function and neuropathological damage after CA/CPR. (A and C) The protein levels of indicated molecules among the sham, vehicle and GLB groups. (B and D) The protein levels of above molecules among the sham, control (treated with the negative control siRNA), and siRNA groups. (E) Confocal analysis of TRPM4 (green) and SUR1 (red) or CD68 (red) staining 12 hours after ROSC. Scale 
bar $=50 \mu \mathrm{m}$. (F) NDSs of survived rats at 24, 48, 72 hours and on day 7 after ROSC. (G and H) The effect of GLB on post-cardiac arrest spatial memory deficits in Morris Water Maze test. (I and $\mathrm{J}$ ) The effect of GLB on post-cardiac arrest neuropathological damage. Scale bar $=100 \mu \mathrm{m}$. Statistical significances are determined with one-way ANOVA followed by Tukey's post hoc test or Mann-Whitney U test (NDSs), except the data of escape latency which are analyzed with repeated-measures ANOVA. Data are presented as mean $\pm S D$, or medians and percentiles (NDSs). ${ }^{*} P<0.05, * \star P<0.01$, ${ }^{\star * *} P<0.001$ versus sham; \#P $<0.05$, \#\#P<0.01, \#\#\# $\mathrm{P}<0.001$ versus vehicle or control. $\mathrm{n}=5$ or 9 per group.

A
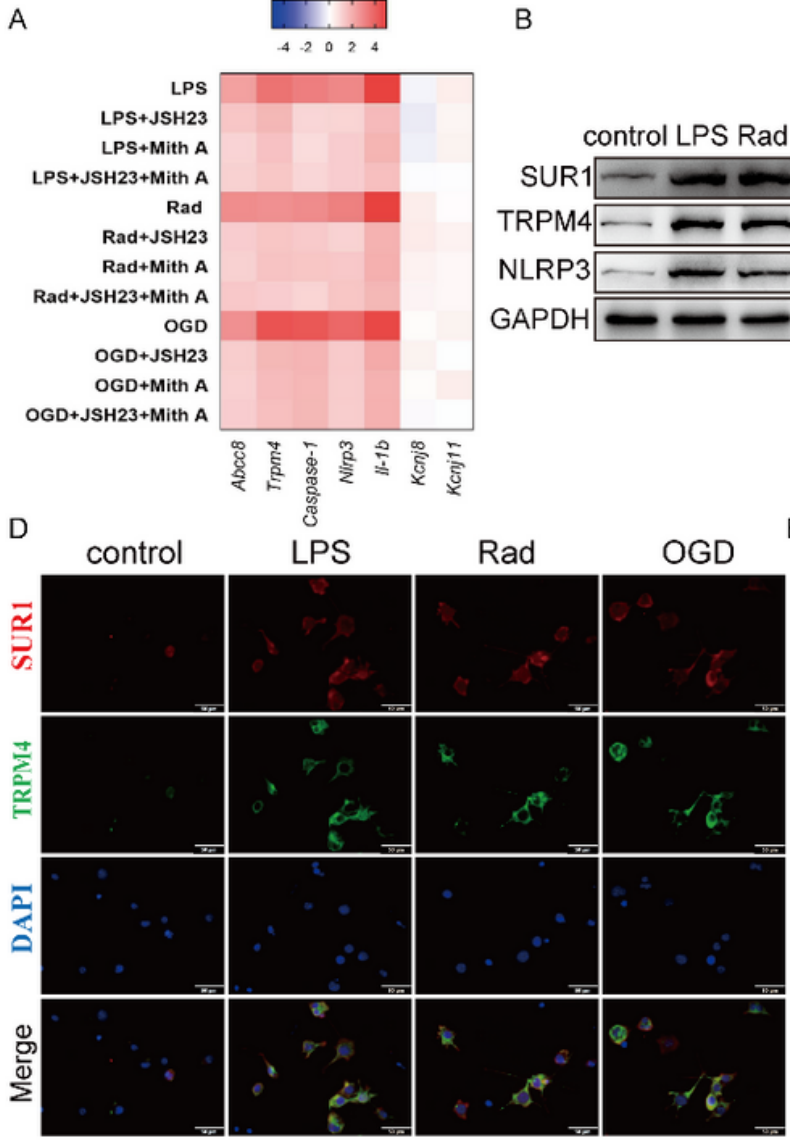

G GAPDH

supernatant IL-1 $1 \beta$
B

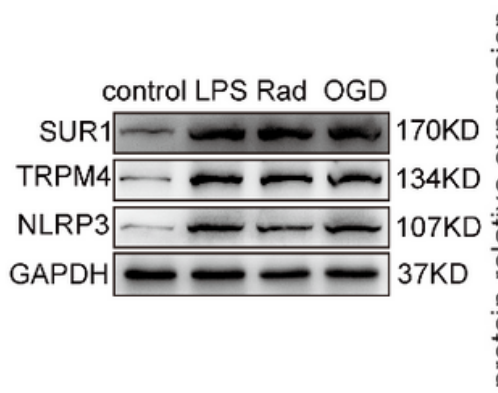

$\mathrm{C}$

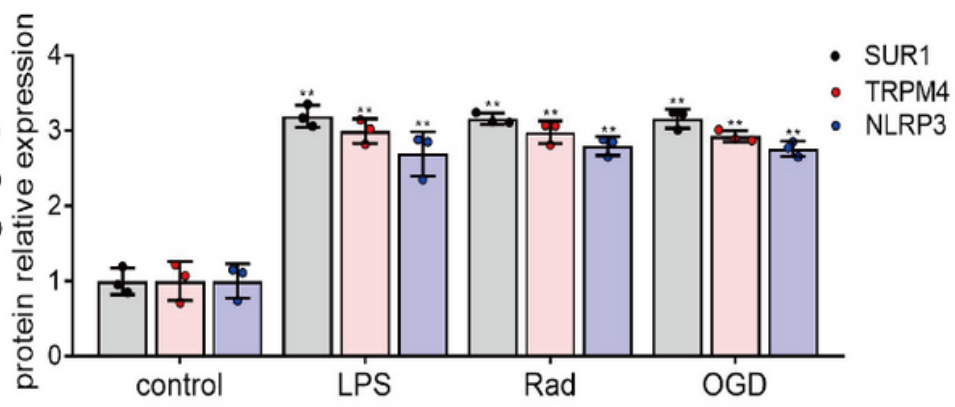

\section{Figure 4}

Assembly of SUR1-TRPM4 complex and NLRP3 inflammasome in BV2 cells challenged with various injurious stimuli. (A) The heatmap reflecting the mRNA levels of indicated genes in LPS-, radiation-, or OGD-primed BV2 cells with or without transcriptional factor inhibitors. Mith A, Mithramycin A. (B and C) Western blotting results of SUR1, TRPM4, and NLRP3 in LPS-, radiation-, or OGD-primed BV2 cells. (D) Confocal analysis of TRPM4 (green) and SUR1 (red) staining in LPS-, radiation-, or OGD-primed BV2 cells. Scale bar $=50 \mu \mathrm{m}$. (E and F) The immunofluorescence reflecting the caspase-1 activity in LPS-, radiation-, or OGD-primed BV2 cells challenged with ATP with or without MCC950. Scale bar $=50 \mu \mathrm{m}$. $(\mathrm{G}$ and H) The 
protein expression levels of caspase-1 p20 and supernatant IL-1 $\beta$ reflecting the NLRP3 activation in radiation- or OGD-primed BV2 cells challenged with ATP with or without MCC950. Rad, radiation. All data in this figure are analyzed using one-way ANOVA followed by Tukey's post hoc test. Data are represented

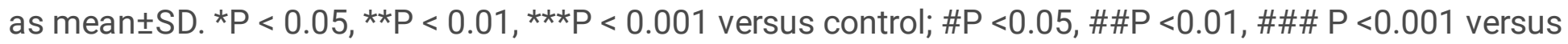
LPS-, radiation- or OGD-primed BV2 cells challenged with ATP. $n=3$ per group.
A
control
LPS+ATP

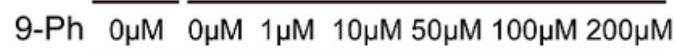

caspase-1 p20

GAPDH

supernatant IL-1 $\beta$

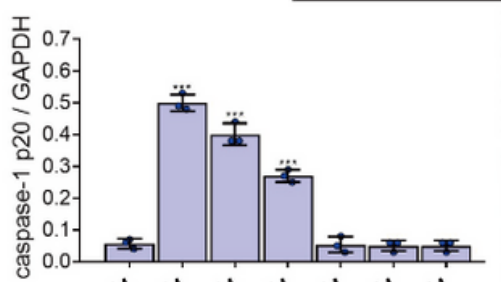

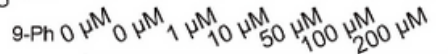

control LPS+ATP
contro

C

GLM $\frac{\text { LPS }+ \text { ATP }}{0 \mu \mathrm{M} 1 \mu \mathrm{M} 10 \mu \mathrm{M} 50 \mu \mathrm{M} 100 \mu \mathrm{M} 200 \mu \mathrm{M}}$

caspase-1 $\mathrm{p} 20 \longrightarrow-\cdots-\cdots$
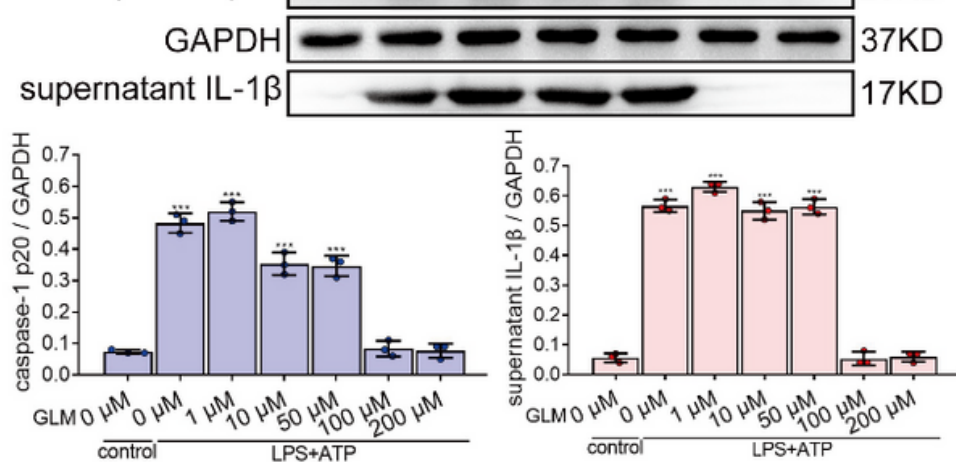

E
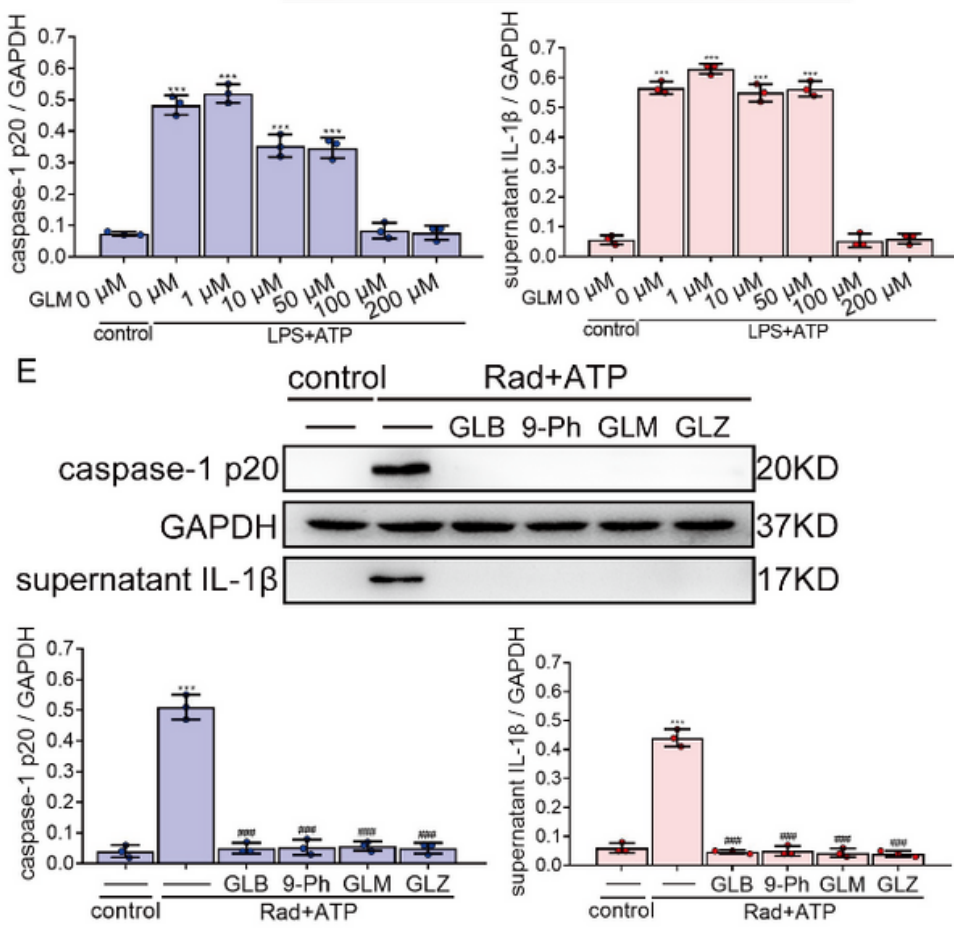

B

control

LPS+ATP

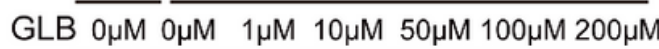

caspase-1 $\mathrm{p} 20$ - $20 \mathrm{KD}$

GAPDH $-\infty$ 37KD

supernatant IL-1 $13 \longrightarrow$ 17KD

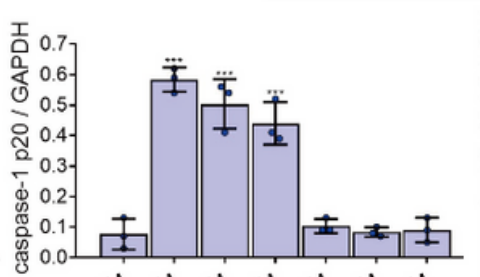

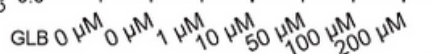
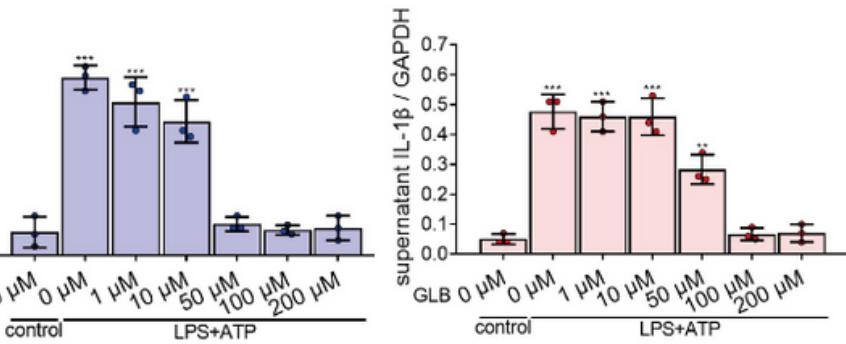

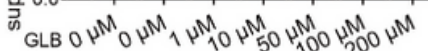

control LPS+ATP

D

control

LPS+ATP
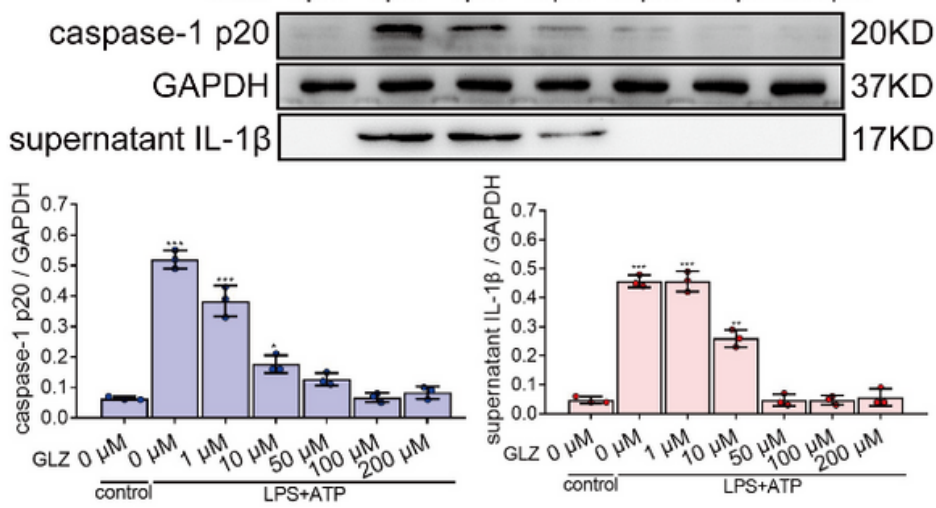

F control OGD+ATP
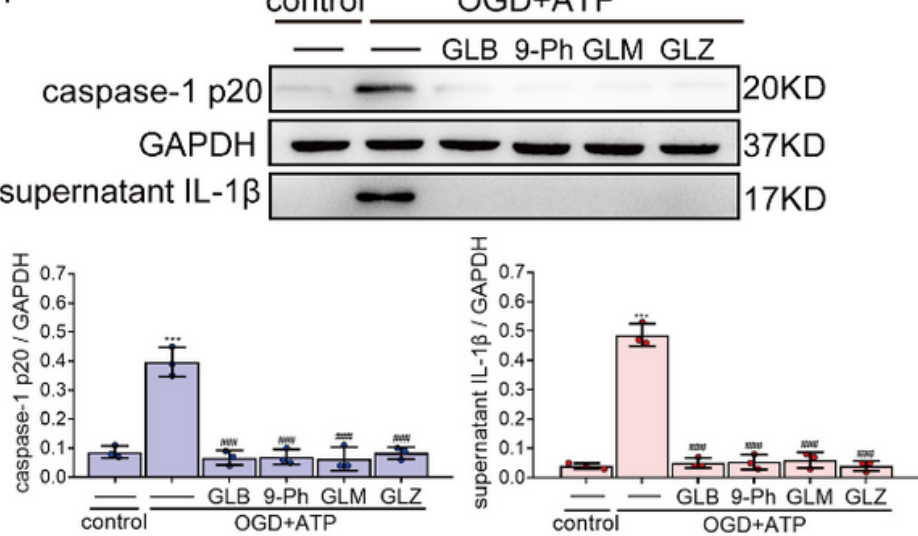

\section{Figure 5}

SUR1-TRPM4 deficiency reverses the activation of NLRP3 inflammasome in BV2 cells. (A-D) Western blotting results manifesting the levels of NLRP3 inflammasome activation in LPS-primed BV2 cells challenged with ATP, when exposed to the concentration gradient of 9-Ph (A), GLB (B), GLM (C), and GLZ 
(D). (E and F) Western blotting results manifesting the levels of NLRP3 inflammasome activation in radiation- or OGD-primed BV2 cells challenged with ATP, in the presence of indicated SUR1 or TRPM4 inhibitors. Rad, radiation. All data in this figure are analyzed using one-way ANOVA followed by Tukey's post hoc test. Data are represented as mean $\pm S D$. ${ }^{*} P<0.05,{ }^{\star *} \mathrm{P}<0.01,{ }^{\star \star *} \mathrm{P}<0.001$ versus control; \#P $<0.05$, \#\#P $<0.01$, \#\#\# $\mathrm{P}<0.001$ versus radiation- or OGD-primed BV2 cells challenged with ATP. $\mathrm{n}=3$ per group.
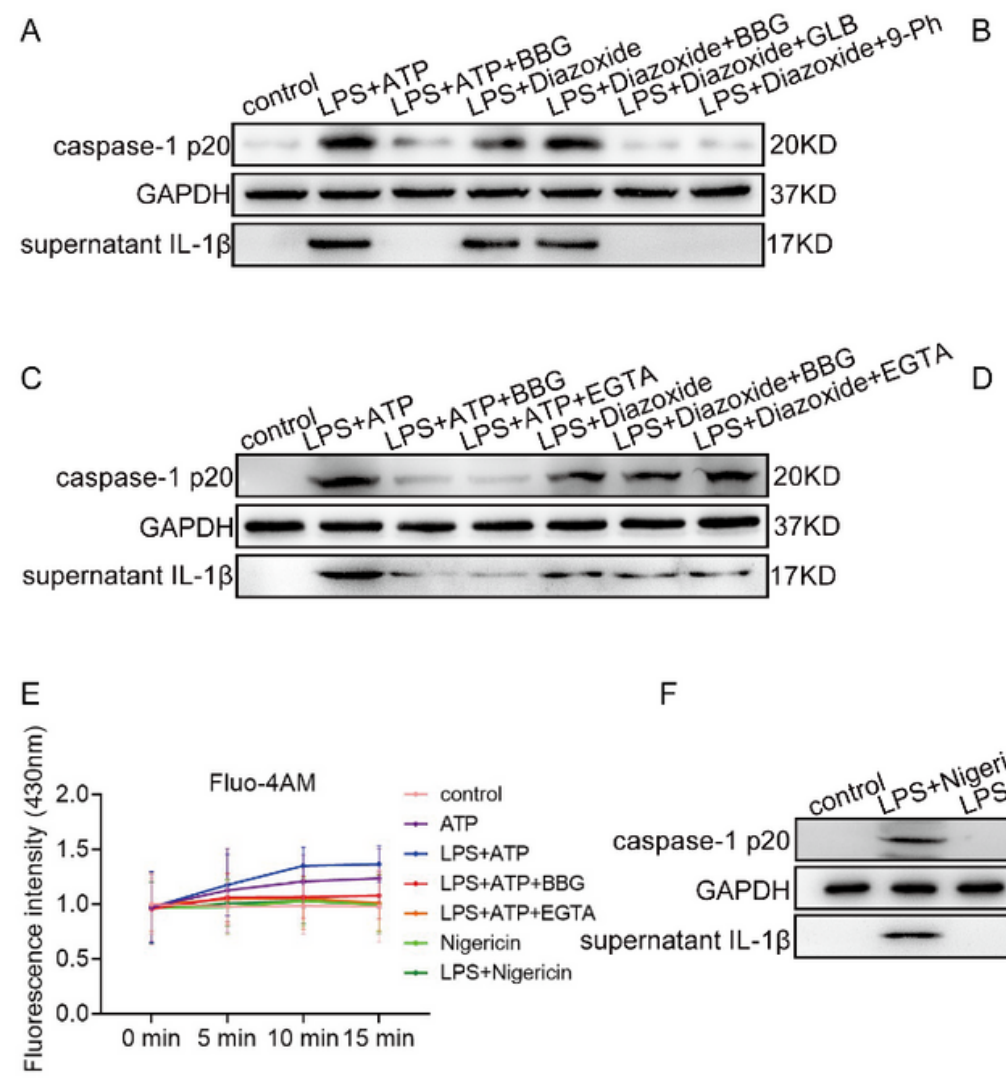

$\mathrm{F}$
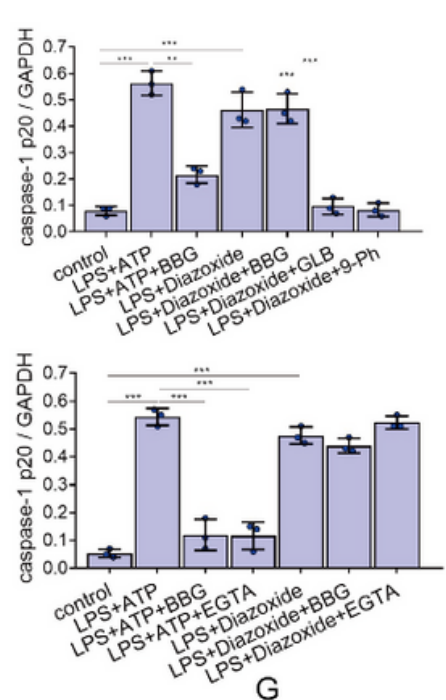
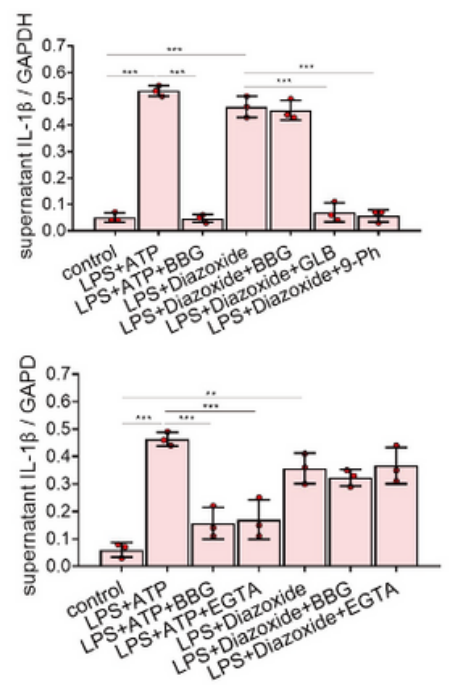

$\mathrm{H}$
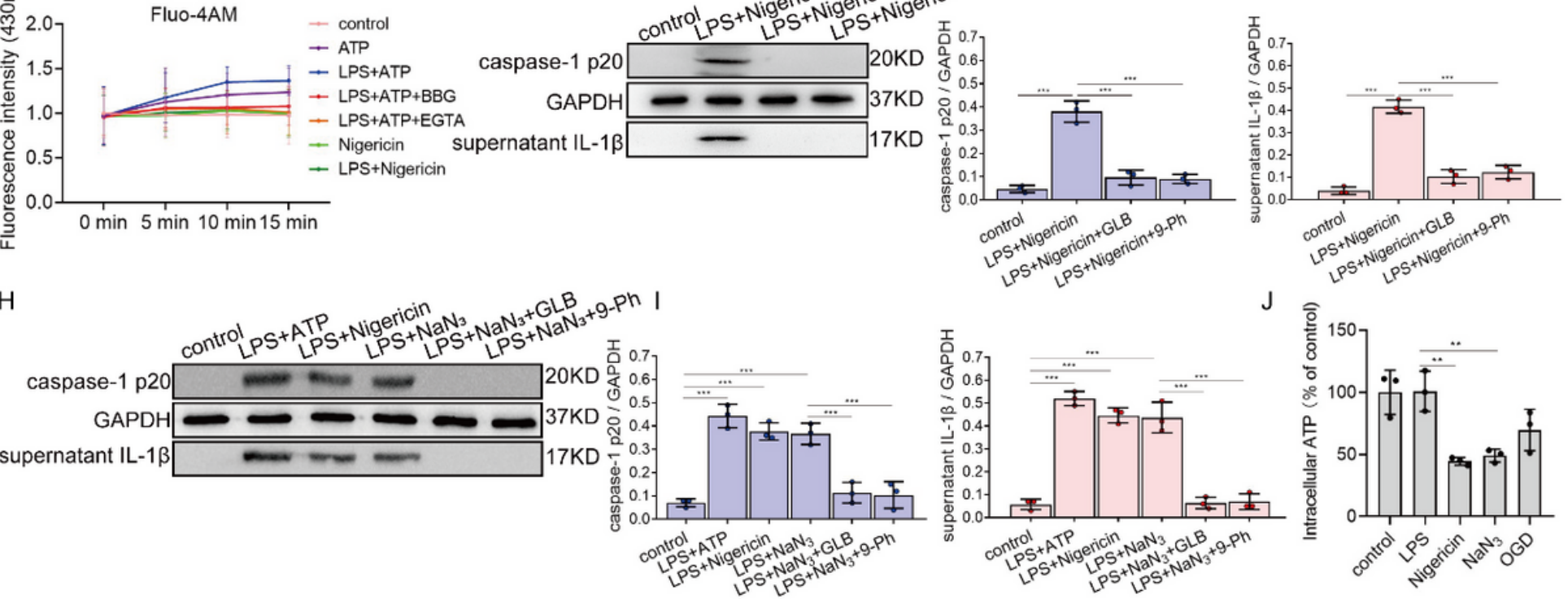

\section{Figure 6}

The activation of SUR1-TRPM4 in BV2 cells involves upstream P2X7R-mediated Ca2+ influx, and participates in the NLRP3 activation mediated by the ATP decrease in BV2 cells. (A-D) Western blotting results reflecting NLRP3 activation in LPS-primed BV2 cells challenged with ATP or diazoxide, in the presence of BBG, GLB, 9-Ph or EGTA. (E) The change in intracellular $\mathrm{Ca} 2+$ of $\mathrm{BV} 2$ cells after various stimulations and interventions. (F-I) Western blotting results reflecting NLRP3 activation in LPS-primed BV2 cells challenged with ATP, nigericin, or NaN3, in the presence of GLB or 9-Ph. (J) The histogram to show the level of intracellular ATP in indicated groups. All data in this figure are analyzed using one-way 


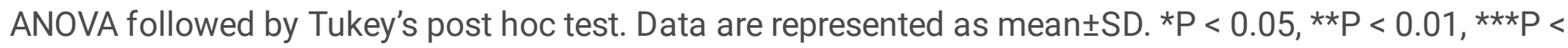
0.001. $n=3$ per group.
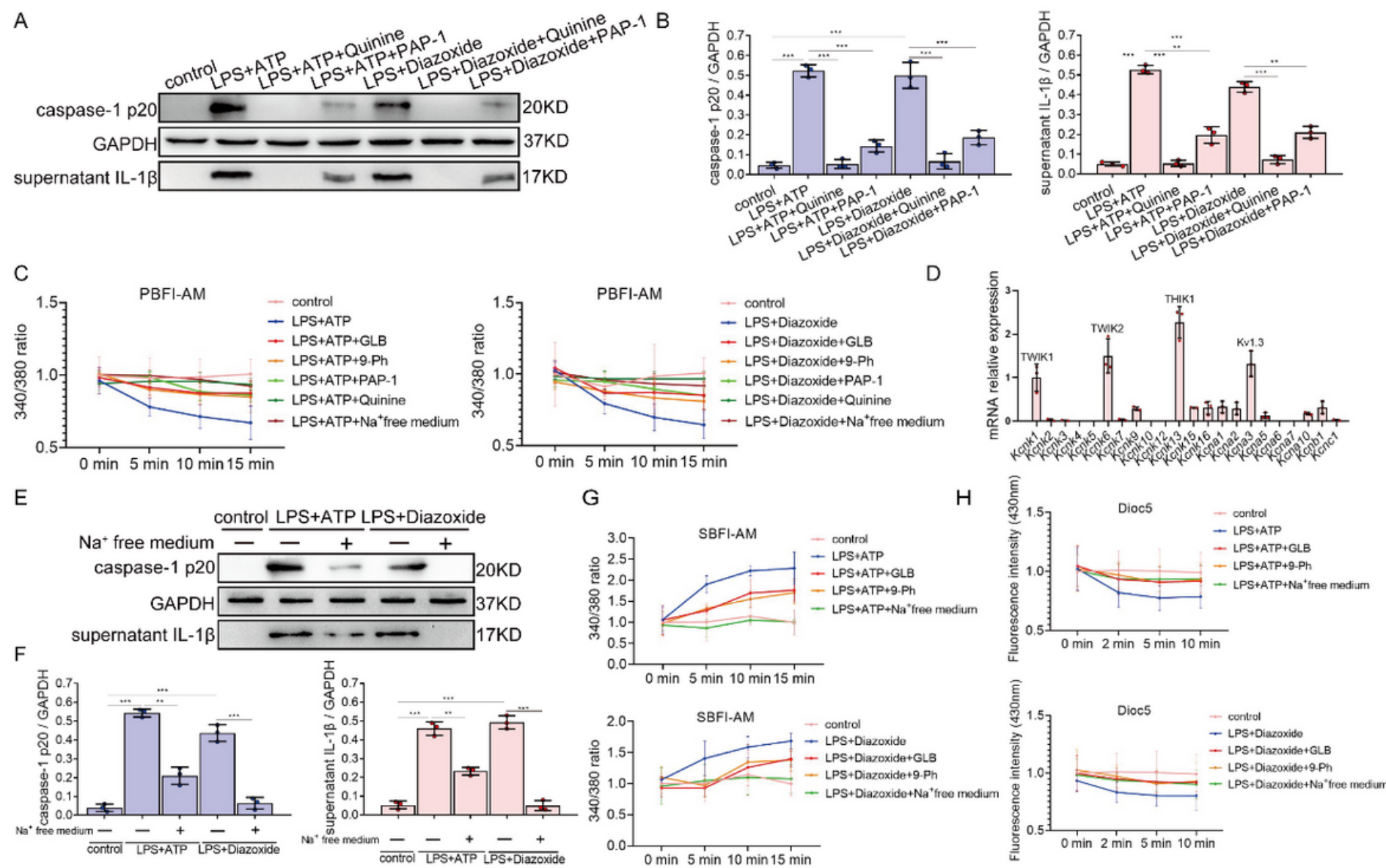

\section{Figure 7}

SUR1-TRPM4 magnifies the $\mathrm{K}+$ efflux via the $\mathrm{Na}+$ influx-driven opening of downstream $\mathrm{K}+$ channels in BV2 cells. (A and B) Western blotting results reflecting NLRP3 activation in LPS-primed BV2 cells challenged with ATP or diazoxide, in the presence of quinine or PAP-1. (C) The change in intracellular K+ of BV2 cells after various stimulations and interventions. (D) qRT-PCR results showing the mRNA levels of representative $\mathrm{K}+$ channels in LPS-primed BV2 cells. (E and F) Western blotting results reflecting NLRP3 activation in LPS-primed BV2 cells challenged with ATP or diazoxide, when bathed in a nominally Na+free medium. (G) The change in intracellular Na+ of BV2 cells after various stimulations and interventions. $(\mathrm{H})$ The change in membrane potential of BV2 cells after various stimulations and interventions. All data in this figure are analyzed using one-way ANOVA followed by Tukey's post hoc test. Data are represented as mean $\pm S D$. ${ }^{*} P<0.05,{ }^{*} \mathrm{P}<0.01,{ }^{*} * \mathrm{P}<0.001 . \mathrm{n}=3$ per group. 


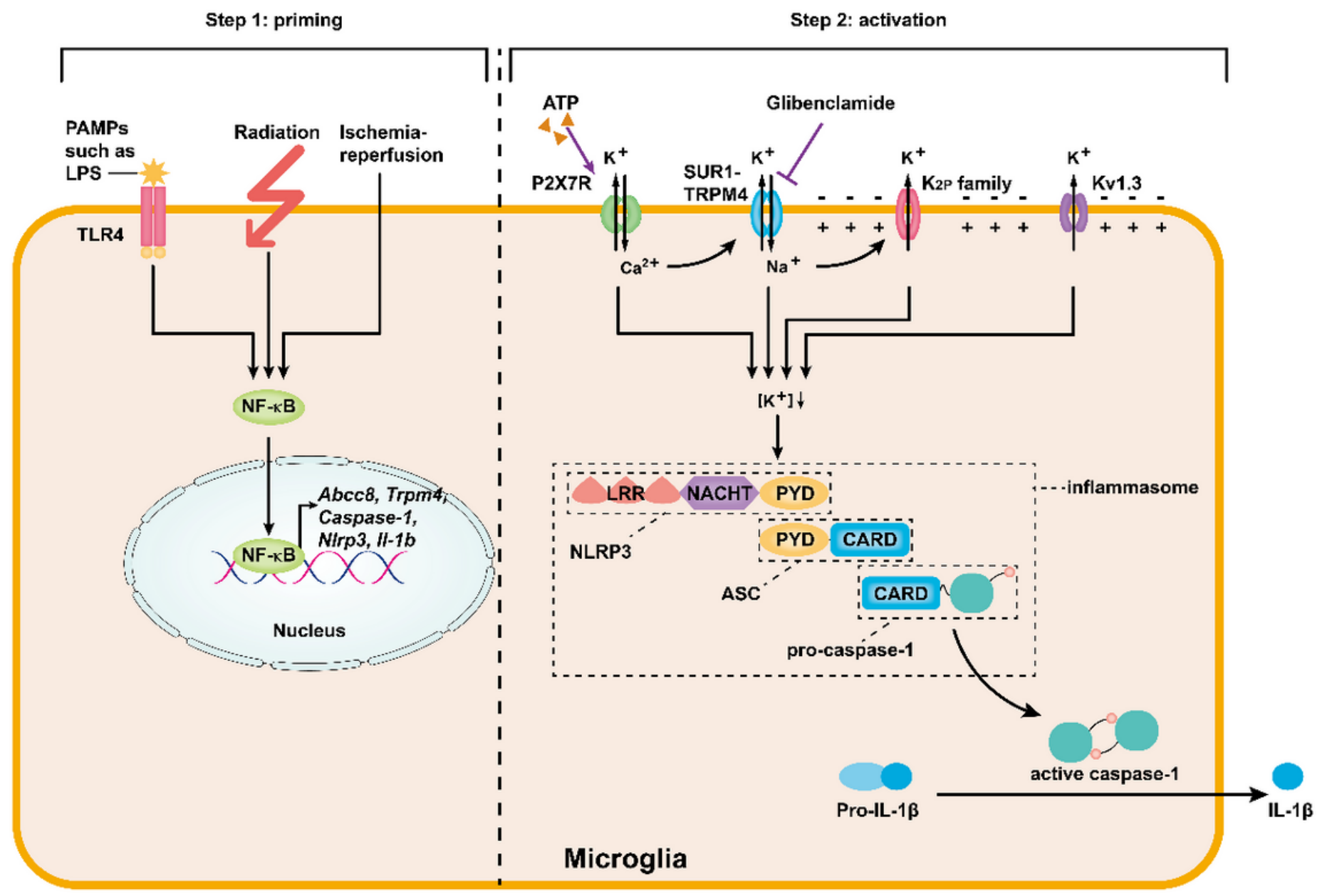

Figure 8

The regulatory mechanism of SUR1-TRPM4 in NLRP3-mediated neuroinflammation. In current study, we have demonstrated that the environment in multiple neurological disorders can provide both the priming signal to induce the NF-KB-dependent transcriptional upregulation of SUR1-TRPM4 complex and NLRP3 inflammasome components, and the activation signal to trigger inflammasome assembly. The signal 1 (priming; left) is provided by the radiation, the ischemia-reperfusion, and the activation of TLR4 with PAMPs such as LPS. After priming, the signal 2 (activation; right) is provided by DAMPs like ATP which binds to P2X7R to activate SUR1-TRPM4 by mediating Ca2+ influx. SUR1-TRPM4 acts as an integrated switch of indicated $\mathrm{K}+$ channels and an amplifier of $\mathrm{K}+$ efflux through mediating $\mathrm{Na}+$ influx and contributing to the depolarized membrane potential, and thereby initiates the NLRP3 inflammasome activation in microglia when the decreased intracellular $\mathrm{K}+$ reaches the threshold to engage NLRP3. Formation of the inflammasome activates caspase- 1 , which in turn processes pro-IL-1 $\beta$ into its mature form. CARD, caspase recruitment domain; LRR, leucine-rich repeat; PAMPs, pathogen-associated molecular patterns; PYD, pyrin domain; TLR4, Toll-like receptor 4.

\section{Supplementary Files}


This is a list of supplementary files associated with this preprint. Click to download.

- supplementarymaterials.docx 